\title{
A Team Study of a Multiple-Power Wireless Random Channel Access Mechanism with Capture Effect
}

\author{
Abdelillah Karouit, ${ }^{1}$ Essaid Sabir, ${ }^{2}$ Fernando Ramirez-Mireles, ${ }^{3}$ \\ Luis Orozco Barbosa, ${ }^{4}$ and Abdelkrim Haqiq ${ }^{1}$ \\ ${ }^{1}$ L-IR2M, Faculty of Sciences and Techniques Hassan 1st University, Settat, Morocco \\ ${ }^{2}$ ENSEM-GREENTIC Hassan II University Casablanca, Morocco \\ ${ }^{3}$ Engineering Division, ITAM, Mexico City, DF, Mexico \\ ${ }^{4}$ University of Castilla La Mancha (UCLM), 02071 Albacete, Spain
}

Correspondence should be addressed to Abdelillah Karouit; akarouit@gmail.com

Received 25 March 2013; Revised 24 June 2013; Accepted 25 June 2013

Academic Editor: Xu Zhang

Copyright (C) 2013 Abdelillah Karouit et al. This is an open access article distributed under the Creative Commons Attribution License, which permits unrestricted use, distribution, and reproduction in any medium, provided the original work is properly cited.

\begin{abstract}
We present a team analysis of a slotted random wireless channel access mechanism. Under the proposed scheme, denoted wireless random access mechanism with multiple power levels (MPL-WRA), each mobile station contends for a transmission opportunity following the principles of a slotted access mechanism incorporating a random transmitting power value selected among various available power levels. In this way, a capture effect may be produced allowing the packet to be decoded whenever the signal-tointerference-plus-noise ratio is higher than a given threshold. In order to analyze the performance and optimization of the proposed setup, we build a Markovian model integrating the wireless access mechanism supplemented by the use of multiple power levels in an attractive and simple cross-layer fashion. We follow a team problem approach allowing us to fine tune the design parameters of the overall system configuration. Throughout an extensive numerical analysis, our main results set the basis for the social optimal system configuration of the proposed mechanism taking into account the physical constraints of using multiple power levels and the actual practical implementation of a slotted access mechanism. We end the paper with concluding remarks and future research directions including guidelines for the actual implementation of our proposal.
\end{abstract}

\section{Introduction}

Despite the huge number of works carried up to date on the performance analysis of various medium access control (MAC) paradigms, random access methods are still the subject of many studies aiming to better understand the effective allocation of the channel. This family of protocols forms an ultimate solution to split intelligence between the base stations and end-user terminals. We believe that random access-based protocols will lead to the design of more robust and efficient protocols in the future. Clearly, modern wireless access network protocols are often based on wireless random access (WRA) mechanisms. The design of such protocols raises novel challenges and difficulties, as wireless networks become more popular. The most studied issue is the channel underutilization imposed by the medium access methods, $37 \%$ when using the slotted Aloha protocol. The basic underlying assumption in legacy WRA protocols is that any concurrent transmission of two or more users causes all transmitted packets to be lost. However, this model does not reflect the actual situation in many practical wireless networks, where some information can still be received correctly even in the case of a simultaneous transmission of two or more packets. Therefore, this feature has recently attracted the attention of many studies spurred by the need of improving the performance of such networks. Many of these studies have shown that the packet transmitted with the strongest power level can still be received successfully (captured) in the presence of contending transmissions if its power level is sufficiently high. It occurs in networks with single packet reception capability, where packets arrive at the common receiver at different power levels due to near-far 
effect, shadowing, or fading. The effect of capture on WRA protocols has been studied extensively in the literature and new MAC protocols for channels with capture have been proposed.

Despite the bounty of works and efforts investigated in analyzing WRA protocols with capture effect, our approach is different. In addition of a capture model based on signalnoise-to interference [1], we consider a random choice of power levels and fine tune the transmit probability in order to maximize an objective function. We start by setting the basis towards the optimization of the main design parameters of the protocol. Towards this end, and following a cooperative game theory approach, we determine the optimal transmission probabilities maximizing the average throughput or minimizing the expected delay. We show that our proposal, namely, MPL-WRA, performs better than a single-power WRA scheme in terms of average throughput, expected delay, and failure probability.

Prior Works. WRA mechanisms have attracted an increasing interest from researchers and practitioners in recent years. In [2], the authors survey, classify, and analyze $34 \mathrm{MAC}$ layer protocols for wireless ad hoc networks, ranging from industry standards to research proposals. A first approach on the analysis of random access protocols is the fundamental theoretical issue of stability. The work in [3] discusses the stability of slotted Aloha with selfish users behavior and perfect information. Authors showed the existence of an equilibrium and characterized it. The earlier works $[4,5]$ study the stability region of slotted Aloha, defined for a given transmit probability vector, as the set of arrival rate vectors for which the system is stable. While in some cases (e.g., [6]), they define the stability region as the set of those arrival rates for which there exists a transmit vector for the Aloha system.

One of the earlier works that analyzed the stability of a WRA incorporating conflict resolution mechanisms is [7]. The mathematical model developed therein showed the instability of the binary exponential back-off (BEB) mechanism, that is, the rate of successful transmissions tends to zero as the load increases. Given the arrival rate, Goodman et al., see [8], attempted to formulate the conditions to guarantee that the delay of the backlog of packets awaiting transmission remains bounded in time. First, they consider a general case of more than two stations sharing the channel, each having an infinite buffer where packets stayed before transmission. However, through simulation results, the authors were only able to show that alternative retransmission protocols (linear backoff) can significantly improve the performance of a network configuration consisting of only two stations. In [9], the authors claim that for any finite number of independent and identical users in the system, and any i.i.d. (independent and identically distributed) arrival process per station, the random access algorithms are stable, provided that the total input rate is less than one. In $[10,11]$, the authors have addressed the optimization problem of the performance of the WRA procedure used by the IEEE 802.16 standards. They have determined the retransmission probabilities and initial window backoff allowing them to optimize the throughput and delay.
Recently, the research community tends to believe that the performance of future networks will be enhanced by the use of a cross-layer design involving the physical, MAC, network and potentially higher layer protocols. Indeed, a crosslayer approach transports feedback dynamically via the layer boundaries to compensate the QoS. In $[12,13]$, authors have introduced a random power level scheme for slotted Aloha. These new algorithms have allowed the system to improve the network throughput and packet delays. However, the capture model considered in [12] is not realistic; authors assume therein that when a unique mobile chooses the highest power, compared to other mobiles, its (re)transmission is successful independently of the power levels used by the other MSs and their respective choices. However, this assumption may not be always true. Indeed, the aggregate signal of other mobiles may jam the signal of the tagged mobile, that is, of the one using the highest power level. A general capture model has been analyzed and considered in [1], where a mobile station (MS) transmits successfully if its instantaneous SINR is higher than the target threshold. The model is validated by Monte Carlo simulation, and the results show that it exhibits better performance results. This potential synergy of information exchange between different layers, instead of the standard OSI noncommunicating layers, has encouraged us to build a simple cross-layer-based WRA procedure.

Other studies have focused on the study under saturation conditions and/or adaptive scheme. For instance, the work in [14] analyzes the WRA and obtains saturation throughput and medium access delay of a packet for a given number of nodes. And in [15], authors provide a framework to study the efficiency of the so-called learning BEB (L-BEB), a version of the WRA that chooses a deterministic back-off value after a successful transmission.

Here, we are interested in the interaction and complementarities of the MAC and physical layers. Thus, we develop an enhanced version of the wireless random access algorithm, where power diversity and a general capture effect are considered. Power diversity has indeed proven its efficiency to improve bandwidth utilization of slotted Alohalike protocols; see [1]. We, thus, expect to obtain a similar trend for random access methods with channel sensing capability. In order to point out the performance benefits, we undertake our study by analyzing the performance of the following two wireless random access mechanisms.

(1) Single power level wireless random access mechanism (WRA): under this mechanism, all mobile stations (MSs) use the same and unique power transmission level. Whenever an MS gets ready to transmit, it attempts to access the channel at the beginning of a time slot. In the case that more than one MS transmits during the same time slot, a channel access conflict arises. In order to resolve the conflict, a simple backoff procedure consisting in the adaptation of the retransmission probability is activated. In this way, not only the MSs involved in the conflict, but also the MSs getting ready to transmit may gain access after several attempts. This procedure is similar in operation to the binary exponential back-off procedure 
used by a large number of IEEE Wireless Communications Standards, such as the IEEE 802.11 among others. We will further develop on the applicability of our results to parameterize the back-off procedure later on.

(2) Multiple power levels wireless random access mechanism (MPL-WRA): the proposed back-off procedure is built based on the aforementioned mechanism, where the competing MSs may take advantage from power diversity. An MS may transmit at a higher power level than the ones used by the other competing stations during the same time slot. In this way, when the instantaneous signal-to-interference-and-noiseratio is higher than a certain threshold, the base station BS may filter other signals considered as noise. It follows that the packet transmitted at a higher power level than any other packet may be successfully extracted and properly decoded.

Following a cooperative game theory approach, we study WRA from a team problem point of view, that is, all mobiles stations optimize the same objective function (maximize the system throughput or minimize the expected delay). Here, we analyze the case when there exists a central entity (base station) that computes the optimal strategy profile and broadcasts it to the operating mobiles. The BS should know the total number of active mobiles and their individual arrival rates. We allow prioritization by incorporating a selective transmit power allocation mechanism. At the beginning of each slot, each mobile picks a power level from the $N$ available power levels and decides to transmit/retransmit its packet.

We start by building a stochastic model to analyze performance of both WRA mechanisms under study. Different to previous studies reported in the literature, the assumption of saturated MSs is relaxed in this paper; then at any instant of time, each MS may be either idle or busy. A Bernoulli process is plausible to model the packet generation.

The rest of the paper is organized as follows. In the next section, we start by describing the models of the two main elements of the system under study: the physical layer and the medium access protocol. In Section 3, we build the Markovian models for the two MAC protocols under study: WRA and MPL-WRA. In Section 4, we derive the performance measures of interest. We perform an in-depth numerical analysis of our results in Section 5. Finally, our concluding remarks are drawn in Section 6.

\section{Protocol Modeling}

For ease of understanding, we summarize the main notation of our model in Table 1.

2.1. Propagation Model and Capture Effect. One of the main contributions of this work consists in the study of power diversity as a means to enhance the efficiency of WRA mechanisms to handle the messages (packets or bandwidth BW requests). Different to previous works reported in the literature following a team problem approach, we conduct a detailed analysis of the number and values of the power levels
TABLE 1: Main notations used in the paper.

\begin{tabular}{|c|c|}
\hline Notation & Description \\
\hline$M$ & The total number of mobile stations. \\
\hline$n$ & $\begin{array}{l}\text { The number of backlogged packets at the beginning } \\
\text { of the current slot. }\end{array}$ \\
\hline$\lambda$ & Packet arrival probability. \\
\hline$q^{i}$ & $\begin{array}{l}\text { Retransmission probability of backlogged packet of } \\
\text { MS } i \text {. }\end{array}$ \\
\hline$P_{n, n^{\prime}}$ & Transition probability from state $n$ to state $n^{\prime}$ \\
\hline$\pi_{n}$ & The steady probability at state $n$. \\
\hline$A_{s}$ & $\begin{array}{l}\text { Probability that a packet transmission succeeds in } \\
\text { the presence of } s \text { simultaneous packet transmission } \\
\text { attempts. }\end{array}$ \\
\hline$W$ & Average window size. \\
\hline$N$ & The number of power levels. \\
\hline$\Theta_{i}$ & SINR of MS $i$ experienced by the base station. \\
\hline$\Theta_{\text {th }}$ & $\begin{array}{l}\text { Target SINR allowing the BS to correctly decode } \\
\text { received packet. }\end{array}$ \\
\hline$T_{j}$ & The $j$ th power level. \\
\hline$x_{j}$ & Probability to select the $j$ th power level. \\
\hline$h_{i}$ & The channel gain of MS $i$. \\
\hline
\end{tabular}

used by the MSs. We further look at the sensitivity threshold of the signal-to-interference-and-noise-ratio (SINR). From now on, we will refer to the data units transmitted by the MSs as packets. In the literature, this term may refer to a control packet with or without data.

Let $T=\left\{T_{1}, T_{2}, \ldots, T_{N}\right\}$ be the power levels available to the MSs to transmit the packets. Time is divided into equal length slots. An MS $i$ attempting to transmit a packet randomly chooses a power level among $N$ available levels. Then, it attempts to access the channel at the beginning of the current slot: (1) if the base station succeeds to decode the signal received from MS $i$ even in the presence of accumulative noise plus interference caused by other adversarial MSs, then the transmission is successful; (2) otherwise, the packet is considered lost and MS $i$ should attempt its retransmission after a random period of time denoted by $W$. In other words, we consider a general capture model, where a packet transmitted by MS $i$ is received successfully when and only when its instantaneous SINR is higher than some given threshold $\Theta_{\text {th }}$. Denote by $\sigma^{2}$ the power of the thermal noise. During a communication between MS $i$ and the BS, the MS $i$ transmits with a power level $T_{i}$. The received power on the BS can be related to the transmitted power by the propagation relation $h_{i} \cdot T_{i}$, where $h_{\mathrm{i}}$ is the channel gain experienced by the base station on that link. The instantaneous SINR of MS $i$ is then given by

$$
\Theta_{i}=\frac{h_{i} \cdot T_{i}}{\sum_{k=1, k \neq i}^{M} h_{k} \cdot T_{k} \cdot \mathbf{1}_{k}+\sigma^{2}}
$$

where $\mathbf{1}_{k}$ is the indicator function of the event that at the current slot, MS $k$ transmits its packet. The power 
levels random selection follows the probability vector $X=$ $\left[x_{1}, x_{2}, \ldots, x_{N}\right]$, where the $j$ th entry $x_{j}$ is the probability to select power level $T_{j}$.

2.2. An MAC/PHY Cross-Layer Design. Contrary to the standard OSI noncommunicating layers, the cross-layer concept, with its potential synergy of information exchange between different layers, is increasingly attracting the attention of the research community. Indeed, many studies of packet delay and loss in various network environments (WLAN, WMAN, ad hoc networks, etc.) using cross layer have been reported in the literature, for example, $[1,16,17]$. Here, we develop a cross-layer design of the optimal retransmission probability integrating the services of the MAC layer and the PHY layer. Using a simple model of a WRA mechanism, whenever a collision (two or more simultaneous transmissions) occurs, all involved packets are corrupted and irretrievably lost; whereas in our setting, even if a collision occurs, the packet transmitted with the highest power has a chance to get correctly decoded when its SINR is higher than the threshold $\Theta_{\text {th }}$.

Without loss of generality, we develop in the following the case of one single ranging subchannel and one single ranging code shared by the M MSs. Furthermore, we build a Markovian model for both the single power WRA and for the scheme making use of power diversity, WRA-MPL. We assume that the retransmission probability remains constant during the retransmission attempts. We take the case of a system consisting of a finite number of $M$ mobile stations that intend to transmit a packet. Each MS $i$ handles a buffer sufficient to store exactly one packet; thus, no new packet is generated by MS $i$ before the successful transmission of the current packet. In fact, this is the preferred mode of operation of some wireless technologies, such as the IEEE 802.16 Standard.

Remark 1. We consider mobiles without buffer, that is, mobiles do not generate new packet till the current one is successfully transmitted. Indeed, quite frequently, one uses the WRA protocol for sporadic transmissions of signaling packets such as packets for making reservation for a dedicated channel for other transmissions (that do not use WRA); see the description of the SPADE on demand transmission protocol for satellite communications in [18]. In the context of signaling, it is natural to assume that a source does not start generating a new signaling packet (e.g., a new reservation) as long as the current signaling packet is not transmitted. In that case, the process of attempts to retransmit a new packet from a source after the previous packet has been successfully transmitted coincides with our no buffer model.

Under the model developed herein, an MS $i$ can be in one of two distinguished states: "I" (idle) or "T" (transmitting). At the beginning of each slot and being in state "I," an MS $i$ has no packet to transmit and does generate a new packet with some probability $\lambda$ (for simplicity we restrict to the case where $\lambda$ is the same for all MSs). MSs at state "I" generate a new packet switch to state "T" at the end of the current slot. Being at state "T," tagged MS $i$ attempts to transmit with probability $q^{i}$, until success. If two or more MSs at state "T" attempt the channel simultaneously, then their packets collide. In the case that the packets could not be properly decoded, then the corresponding MSs immediately return to state "T." All corrupted messages get backlogged and are retransmitted after some random time, whereas if exactly one user attempts a transmission from state "T" (WRA) or if the SINR of the received signal is higher than the target (MPL-WRA), then the transmission is successful, and the corresponding user jumps to state "I." The determination of the above random time can be considered as a stochastic control problem. The information structure, however, is not a classical one: the MSs do not have knowledge of the full state information. They do not know the number of backlogged MSs, nor do they know the number of packets involved in a collision. We study this control problem as a team problem, where there is a common goal to all MSs in the network: to maximize the network throughput or to minimize the transmission delay.

We consider the number of backlogged packets (denoted by $n$ ) at the beginning of a slot as state of the system. For any choice of values $q^{i} \in(0,1]$, the state process is a Markov chain that contains a single ergodic subchain (and possibly transient states as well). Indeed, it is easy to check that, conditioning on the actual state of the system, the future and the past are mutually independent (Markov property), specifically, $P\left(n_{k}=u_{k} \mid n_{k-1}=u_{k-1}, n_{k-2}=\right.$ $\left.u_{k-2}, \ldots, n_{0}=u_{0}\right)=P\left(n_{k}=u_{k} \mid n_{k-1}=u_{k-1}\right)$, where $u_{0}, u_{1}, \ldots, u_{k} \in\{0,1, \ldots, M\}$. The other properties will be discussed in Section 4. Define $\overline{\mathbf{q}}$ to be the vector of retransmission probabilities for all mobile stations (whose $i$ th entry is $q^{i}$ ). We note the transition matrix of the Markov chain by $P(\overline{\mathbf{q}})$. Let $\bar{\pi}(\overline{\mathbf{q}})$ be the corresponding vector of the steady-state probabilities, where its $n$th entry $\pi_{n}(\overline{\mathbf{q}})$ denotes the probability that the state of the system is $n$ (number of backlogged mobiles). When all entries of $\overline{\mathbf{q}}$ are the same, say $q$, we will write (with some abuse of notation) $\bar{\pi}(q)$ instead of $\bar{\pi}(\overline{\mathbf{q}})$.

Remark 2. Figure 1 shows that whenever the number of backlogged packets is greater than $0, n>0$, the number of backlogged packets can decrease at most by one and increase at most by $M-n$ per transition.

We restrict in our team problem to simple policies in which $q^{i}$ does not change in time. Since sources are symmetric, we will further restrict the problem to finding a symmetric optimal solution in which the retransmission probabilities $q^{i}$ do not depend on $i$. The WRA protocol is usually built for medium access or sporadic data transfer; the assumption of symmetric sources is then justified.

Remark 3. We restrict to the symmetric solutions due to the fact that if mobile users are asymmetric, then the computation of the system steady state becomes intractable, and consequently the evaluation of throughput and delay will not be possible. Also studying an asymmetric network numerically requires one to consider all possible combinations of the network parameters. Since the degree of freedom (the parameters to choose) is usually very large in 


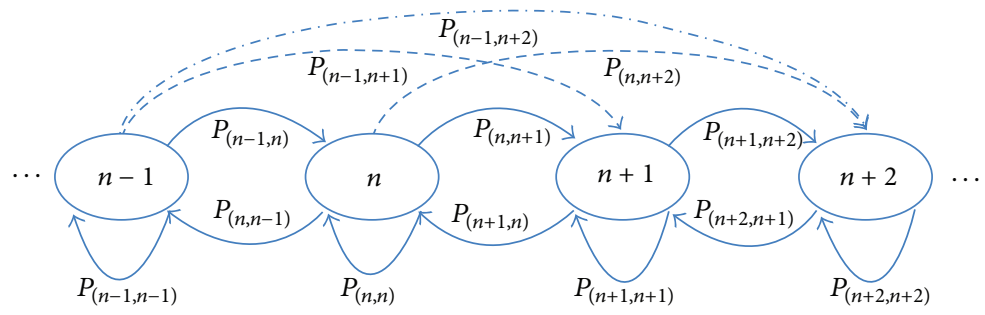

FIGURE 1: State transition diagram.

asymmetric networks, such a numerical study is not carried out generally.

Remark 4. Our WRA model fits well the IEEE 802.16, where quite frequently one uses the binary exponential back-off (WRA) procedure for making a channel reservation for other transmissions (that do not use WRA) or to initiate a handover process. In the context of $\mathrm{BW}$ request, it is natural to assume that a mobile station does not start generating a new signaling message (e.g., a new reservation) as long as the current signaling message is not transmitted. In that case, the process of attempts to start a new BW request from an MS after the previous BW request that has been successfully transmitted coincides well with our one packet buffer model.

Assume that there are $n$ actual backlogged packets and all use the same value $q$ as retransmission probability. Let $Q_{r}(j, n)$ be the probability that $j$ out of the $n(n=0,1, \ldots, M)$ backlogged packets are retransmitted in the current slot. Then,

$$
Q_{r}(j, n)=\left(\begin{array}{c}
n \\
j
\end{array}\right)(1-q)^{n-j}(q)^{j}
$$

Similarly, let $Q_{a}(j, n)$ denote the probability that $j$ unbacklogged MSs generate new BW requests in the current slot. Thus,

$$
Q_{a}(j, n)=\left(\begin{array}{c}
M-n \\
j
\end{array}\right)(1-\lambda)^{M-n-j}(\lambda)^{j}
$$

\section{Markovian Models}

For simplicity and without loss of generality, we restrict in our analysis to the case of a single ranging subchannel. Nevertheless, our analysis is still applicable and easily extendable to the case of several subchannels and several ranging codes.

3.1. Wireless Random Access (WRA) Mechanism. Let us consider the standard WRA algorithm, where all MSs use the same power to transmit their respective packets. The possible transitions of the Markov chain, depicted in Figure 1, can be listed as follows.

(i) Since no more than one MS can succeed a packet in a given slot, then a transition from state $n$ to state $n+k$ with $k<-1$ is impossible. It follows that $P_{n, n+k}=0$, $k<-1$. (ii) A transition from state $n$ to state $n-1$ indicates a reduction in the backlog which is possible only if a single backlogged packet was transmitted. In addition, no new packet should be generated during the current slot.

(iii) The fact that the system stays in state $n$ can be induced by two distinct events. The first event corresponds to the case when no new packet is generated and either there is zero or more than one transmission attempt. The second event inducing this transition results from a situation in which one of the backlogged MSs attempts retransmission and exactly one packet arrival.

(iv) The last case is the transition from state $n$ to state $n+k$ with $1 \leq k \leq M-n$. This can happen when one backlogged MS attempts transmission, while $k+1$ idle MSs generate new packets. Alternatively, the intended transition can be induced when new $k$ packets are generated and there are no departures.

The above observations are summarized in the following transition matrix:

$$
\begin{aligned}
& P_{(n, n+k)}(q)\left\{\begin{array}{rr}
Q_{a}(k, n)\left[1-Q_{r}(1, n)\right], \quad k=M-n, k>1, \\
Q_{a}(k, n)\left[1-Q_{r}(1, n)\right]+Q_{a}(k+1, n) Q_{r}(1, n), \\
1 \leq k<M-n,
\end{array}\right. \\
& Q_{a}(0, n)\left[1-Q_{r}(1, n)\right]+Q_{a}(1, n) Q_{r}(1, n), \\
& \begin{array}{lr}
k=0, \\
Q_{a}(0, n) Q_{r}(1, n), & k=-1,
\end{array}
\end{aligned}
$$

3.2. Multiple Power Levels WRA (MPL-WRA). We now turn to build the Markovian process of the MPL-WRA. Before starting transmission, each MS picks randomly a power level among the $N$ available levels. We highlight here that even if a collision may occur, the BS may be able to decode the packet transmitted at the highest power among all the concurrent transmissions. In fact, all concurrent packets are treated as interfering noise and they are lost, whereas the packet with the highest power is successfully received given that its SINR is higher than the target SINR. In the case when 
all MSs attempt the channel with the same probability $q$, the transition probabilities are then given as follows:

$$
\begin{aligned}
& P_{(n, n+k)}(q)
\end{aligned}
$$

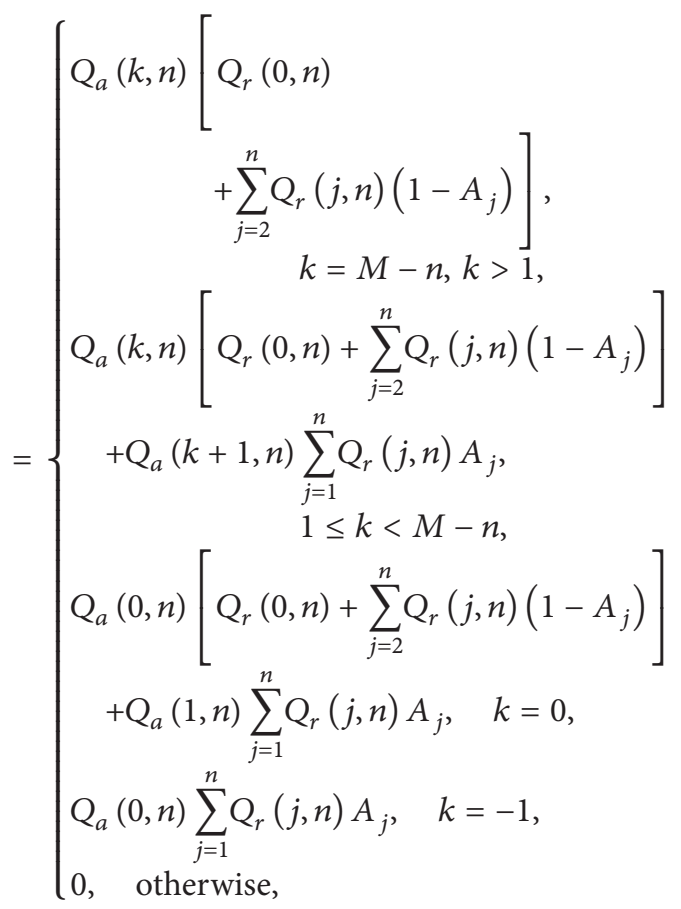

where $A_{s}, s \geq 2$, is the probability of a successful packet among $s$ simultaneous packet transmissions, that is, transmitted during the same symbol/slot. Let us denote by $a_{i}^{s}$ the event that the transmission of the tagged MS $i$ using the strongest power level is successful in the presence of $s-1$ simultaneous transmission attempts. The $A_{s}$ is derived using the following events decomposition $a_{i}^{s}=\sum_{t=2}^{N} P\left(A_{i, s, t} \bigcap B_{i, s, t} \cap C_{i, s, t}\right)$, where

(i) event $A_{i, s, t}$ is "mobile station $i$ attempts transmission with power level $T_{t}$;"

(ii) event $B_{i, s, t}$ is "other $s-1$ mobile stations transmit with power levels lower than $T_{t} ;$ "

(iii) event $C_{i, s, t}$ is "instantaneous SINR of tagged mobile station $i$ is higher than the target SINR $\Theta_{\mathrm{th}}$ ".

We assume that all MSs are symmetric and are assumed to experience the same channel gain, that is, $h_{i}=h, i=$ $1, \ldots, M$. Then if $A_{s}=\sum_{i=1}^{s} a_{i}^{s}=s \cdot a_{i}^{s}$, it follows that

$$
\begin{aligned}
A_{s}= & s \sum_{l=0}^{N-2} \sum_{s_{1}=0}^{s-1} \cdots \sum_{s_{N-l-1}=0}^{s-1} x_{1}^{s_{1}} \cdot x_{2}^{s_{2}} \cdots x_{N-l-1}^{s_{N-l-1}} \cdot x_{N-l}^{s_{1}} \cdot u \\
& \times\left(\frac{T_{N-l}}{\sum_{r=1}^{N-l-1} T_{r} s_{r}+\sigma^{2} / h}-\Theta_{\mathrm{th}}\right) \\
& \cdot \delta\left(s-1-\sum_{r=1}^{N-l-1} s_{r}\right)
\end{aligned}
$$

with $A_{0}=0$ and $A_{1}=1$. The $x_{k}$ is the probability that an MS (with new arrival or backlogged packet) (re)transmits using power level $T_{k} \cdot T_{N-l}$ is the power level chosen by the MS whose transmission may potentially succeed; that is, it corresponds to the highest power selected in the current slot, whereas $s_{k}$ denotes the number of MSs that have chosen the power level $T_{k}$ in the current slot.

$\delta(t)$ is the Dirac distribution and $u(t)$ is the Heaviside function (unit step function), and, are given by (7) and (8), respectively,

$$
\begin{aligned}
& \delta(t)= \begin{cases}1, & t=1, \\
0, & \text { else }\end{cases} \\
& u(t)= \begin{cases}1, & t \geq 0, \\
0, & \text { else }\end{cases}
\end{aligned}
$$

Computing the success probability is a very high time consuming task. The difficulty in formula (6) is to consider one single transmitting MS at the highest power level and to list all the cases where the $s-1$ remaining MSs transmit at lower power levels. This corresponds exactly to the set of partitions (a partition of a positive integer $n$ is a way of writing $n$ as a sum of positive integers). of the positive integer $k-1$ considering all possible permutations. Generating all the partitions of an integer is widely studied in the literature and several algorithms have been proposed, for example, see $[19,20]$. The computational complexity of such algorithms is very expensive and may take a long time to compute the set of all partitions and their permutations. Fortunately, in our model, the success probability depends on one of the following: the instantaneous backlog of the system $n$; the arrival probability $\lambda$; and the retransmission probability $q$. Henceforth, the success probability matrix $\mathbf{A}=\left(A_{s}\right), s=$ $1, \ldots, M$ can be computed once and reused to derive the transition matrix.

\section{Steady State and Performance Evaluation}

Based on the steady state of the system, one can estimate several performance measures. For instance, we are particularly interested in deriving the average throughput, the expected delay, and the failure probability of bandwidth requests. We first discuss how to obtain the steady state probabilities. Then, we solve the optimization problem. We then derive the expressions of the performance metrics of interest functions of the steady state equations. Second, we discuss how to solve the optimization problem and discuss the existence of an optimal solution.

4.1. Performance Metrics. The steady state of the Markovian process is given by the following system:

$$
\begin{gathered}
\bar{\pi}(q)=\bar{\pi}(q) P(q), \\
\pi_{n}(q) \geq 0, \quad n=0, \ldots, M, \\
\sum_{n=0}^{M} \pi_{n}(q)=1 .
\end{gathered}
$$


Using a simple iterative method, one may compute the stationary distribution from the system (9). Hence, the mean number of the bandwidth requests in the system is given by

$$
B(q)=\sum_{n=0}^{M} \pi_{n}(q) n
$$

Similarly, the average throughput, defined as the sample average of the number of packets that are successfully transmitted, is given by

$$
\Gamma(q)= \begin{cases}\sum_{n=0}^{M} \pi_{n}(q) Q_{r}(1, n), & \text { WRA, } \\ \sum_{n=0}^{M} \sum_{j=1}^{n} \pi_{n}(q) Q_{r}(j, n) A_{j}, & \text { MPL-WRA. }\end{cases}
$$

The expected delay of a packet is defined as the average number of slots that a packet takes from a tagged MS to the BS. We denote it by $D(q)$. The average number of packets in the system is given by $B(q)$. In addition, from Little's theorem, the mean sojourn time that each packet spends in the queue is given by the ratio between the average number of packets to their mean departure rate

$$
D(q)=1+\frac{B(q)}{\Gamma(q)} .
$$

The delay is one slot larger, since a packet is assumed to join the system only after the slot in which the packet is generated.

The failure probability of both schemes has as a main source of error the probability of collision. In the case of the single power WRA, the simultaneous attempts of two or more stations results unavoidable on the inability of the BS to decode any of the transmitted packets. However, in the case of the WRA-MPL mechanism, the BS may be unable to decode correctly the received packet transmitted at the highest power level (packets transmitted at lower power levels are systematically corrupted) due to the accumulative noise plus interference. Accordingly, the failure probability of a packet is given by:

$$
\psi(q)= \begin{cases}1-\sum_{n=0}^{M} Q_{r}(1, n) \pi_{n}, & \text { WRA } \\ \sum_{n=0}^{M} \sum_{j=1}^{n} Q_{r}(j, n)\left[1-A_{j}\right] \pi_{n}, & \text { MPL-WRA. }\end{cases}
$$

4.2. Finding the Social Optimum. The optimal solution of the team problem is obtained by resolving the following optimization problem:

$$
\begin{array}{ll}
\max _{q} & \text { objective }(q) \\
\text { s.t. } & \left\{\begin{array}{l}
\bar{\pi}(q)=\bar{\pi}(q) \cdot P(q), \\
\sum_{n=0}^{M} \pi_{n}(q)=1, \\
\pi_{n}(q) \geq 0, \quad n=0,1, \ldots, M,
\end{array}\right.
\end{array}
$$

where objective $(q)$ is replaced by the average throughput or minus expected delay. We note that the solution can be obtained by computing recursively the steady-state probabilities, like problem 4.1 in [18].

Singularity at $q=0$. The only point where the Markov chain does not have a single stationary distribution is at $q=0$, where it has two absorbing states: $n=M$ and $n=M-1$. All remaining states are transient (for any $\lambda>0$ ), and the probability to end in one of the absorbing states depends on the initial distribution of the Markov chain. We note that if the state $M-1$ is reached, then the throughput is $\lambda$. However, if the state $M$ is reached, then the throughput equals 0 , which means that it is a deadlock state. For $\lambda>0$ and $q=0$, the deadlock state is reached with a positive probability from any initial state other than the absorbing state $M-1$. We will therefore exclude the case of $q=0$ and optimize only on the range $\epsilon<q \leq 1$.

Existence of a Solution. The steady-state probabilities $\bar{\pi}(q)$ are continuous over $0<q \leq 1$. This is not a closed interval; therefore, a solution does not necessarily exist. However, as we restrict to the closed interval $[\epsilon, 1]$, where $\epsilon>0$, an optimal solution indeed exists. Therefore, for any $\delta>0$, there exists some $q^{*}>0$ which is $\delta$-optimal. $q>0$ is said to be $\delta$ optimal for the average throughput maximization if it satisfies $\Gamma\left(q^{*}\right) \geq \Gamma(q)-\delta$ for all $q \in[\epsilon, 1]$. A similar definition holds for any objective function (e.g., average delay minimization).

4.3. Back-Off Mechanism. As already stated, WRA mechanisms are widely used by various wireless technologies. In order to solve conflicts arising whenever a collision overcomes, the use of the binary exponential back-off (BEB) algorithms is incorporated into the MAC protocol. In BEB, when an MS tends to contend for a transmission opportunity, it enters a back-off procedure. Whenever the channel is sensed busy, the MS defers the transmission of its message and waits for an idle channel opportunity. In addition and in order to reduce the collision probability due to two or more simultaneous transmissions, an MS is forced to wait for a random number of time slots according to a retransmission probability that depends on the network load conditions. When a message is finally transmitted, the MS has to wait for an acknowledgement ACK, either implicit or explicit ACK depending on the particular network technology being employed. If no notification is obtained in a certain number of subsequent slots, the message is considered lost and a retransmission has to be rescheduled. When the number 
of retransmissions expires, the concerned mobile station discards the packet.

Based on the simple model developed herein, we will make use of our results to determine the average window size, denoted by $W$. At steady state, we assume that the average back-off period corresponds exactly to the average $W$ length while observing the system for a given duration long enough to claim that the stationary regime is met; hence, the average transmission probability is $1 / W$.

Thus, the average window size is given by

$$
W=\frac{1}{q} .
$$

\section{Numerical Investigation}

In this section, we undertake the numerical evaluation of the team problem for the two mechanisms. One of our main goals is to determine the power diversity parameters as well as the optimum retransmission probability by solving the team optimization problem (14). We will further use relation (15) to determine the optimum window size for a given number of active stations.

Throughout our study, we consider a symmetric system; that is, all MSs use the same retransmission probability. We fix $\epsilon=10^{-6}$; that is, the solution for $q^{*}$ will be searched in the closed interval $[\epsilon, 1]$. Throughout our study, we set $\sigma^{2}$ to zero, since the actual value will very much depend on the particular environment of operation and conditions, for example, indoor/outdoor, weather conditions. First, we undertake an in-depth performance evaluation of the MPLWRA mechanism by varying its various design parameters, namely, the target SINR $\Theta_{\text {th }}$, the number magnitudes, and selection policies of the power levels. Once we determine the optimal configuration of the MPL-WRA parameters, we compare the system performance of both protocols, in terms of network throughput and delay by setting the optimal retransmission probability $q$ and average window size $W$, derived from the first part of our numerical analysis. Finally, we study the achievable capacity as function of the number of mobile stations.

5.1. Optimal Configuration of $M P L-W R A$. First, we turn to investigate the impact of the number of power levels, the target SINR $\Theta_{\text {th }}$, and the power levels selection distribution. In the following, we set $M=4$, except otherwise indicated. This will allow to focus on setting the best possible power level parameters: number and selection policies. Since the number of MSs is limited to a small number, we will be in better position to evaluate the impact of the various power parameters on the system performance. Thereafter, we will explore the performance of MPL-WRA by varying the number of MSs.

5.1.1. Power Levels Setting. We first undertake the performance evaluation of the proposed scheme by varying the number and selection policies of the power levels. Our main purpose is to gain an insight on the implementation guidelines of MPL-WRA. Figures 2 and 3 show the retransmission

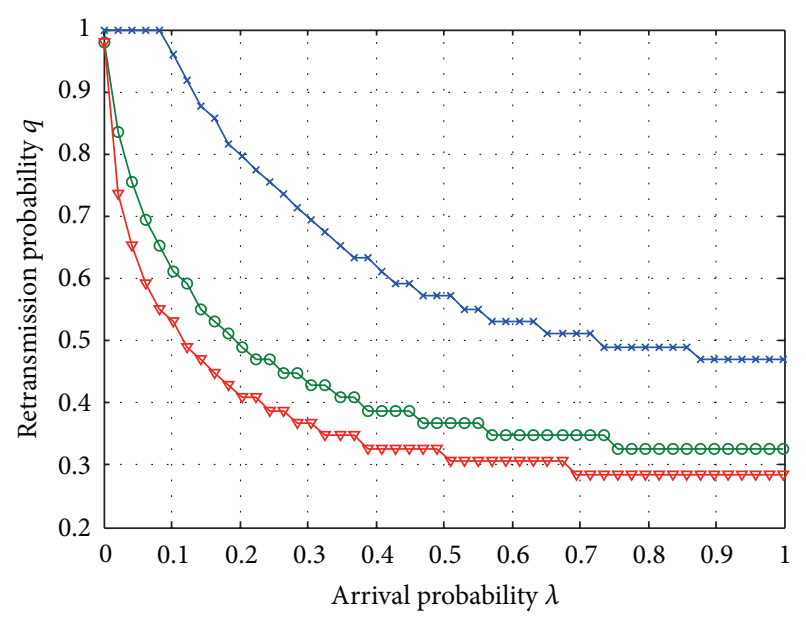

(a)

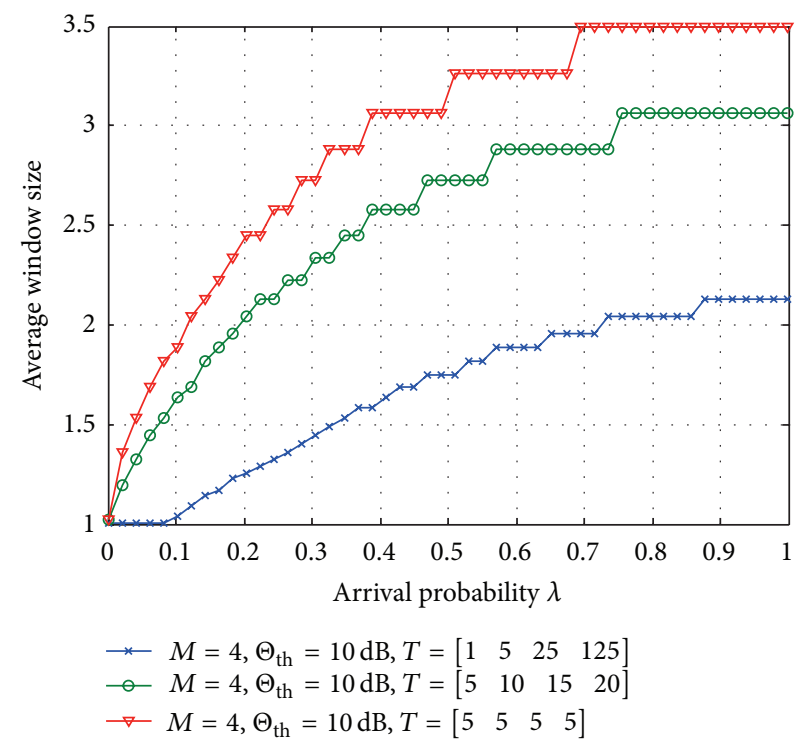

(b)

FIGURE 2: Impact of the set of available power levels on optimal retransmission probability and average window size for $M=4$ mobile stations.

probability and the average window size, and the average throughput and the expected delay, respectively, as function of the arrival probability when changing both the number of values and the distribution of the power levels, using a threshold $\Theta_{\text {th }}=10 \mathrm{~dB}$, and power levels with uniform distribution probability.

We notice that the best performance results are obtained when the power levels follow a geometric progression, whereas the same power scenario provides the lowest performance. The results can be simply explained by the difference between the power levels and its strong impact on the instantaneous SINR value. For a tagged MS $i$, with geometric progression, only adjacent power levels may interfere with the power having been selected $T_{l}^{i}$, whereas the use of an arithmetic progression when setting the power levels will result in a significantly higher interference probability. 


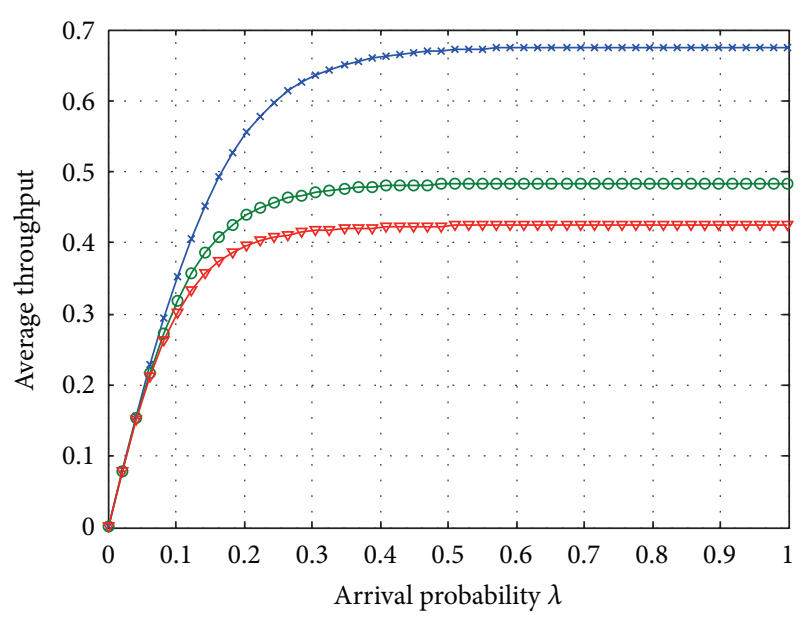

(a)

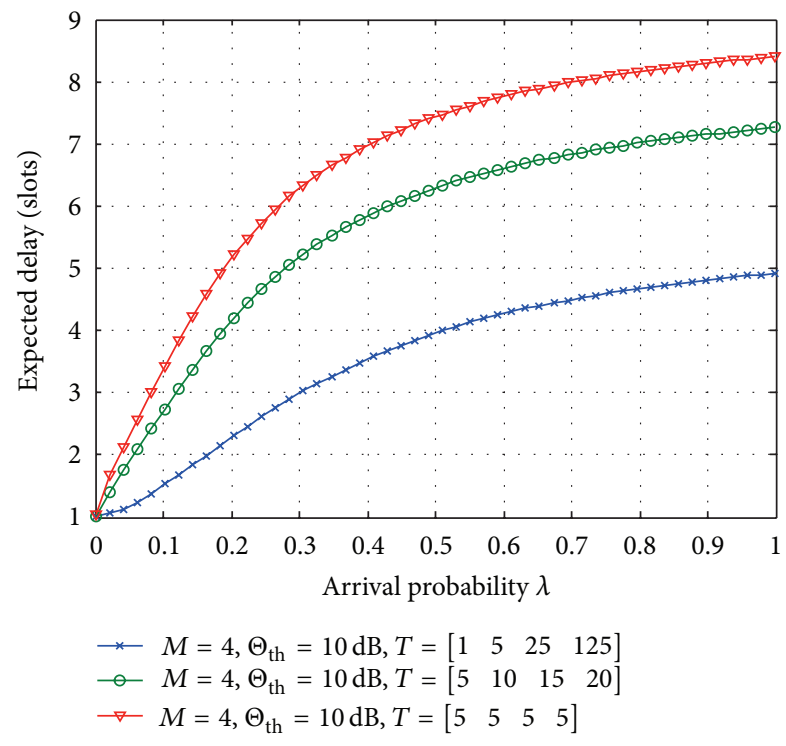

(b)

Figure 3: Impact of the set of available power levels on average throughput and expected delay for $M=4$ mobile stations.

The worst case and equivalent to the use of the single power WRA scheme is given when setting the four power levels to the same value.

5.1.2. Number of Power Levels. We now focus on analyzing the number of actual power levels to be used and its benefits on the performance of the proposed scheme. We consider therefore the use of two, three, or four power levels with their values set following a geometric progression. The power levels for the various configurations have been set as follows: $T=[1,5] \mathrm{mW}(N=2), T=[1,5,25] \mathrm{mW}(N=3)$, and $T=[1,5,25,125] \mathrm{mW}(N=4)$ following a uniform selection policy; that is, a power level is equally probable to be selected by an MS. We have set the threshold $\Theta_{\text {th }}=10 \mathrm{~dB}$.

Figures 4 and 5 show the variation of retransmission probabilities, the average window size, the average throughput, and the expected delay, respectively, as function of

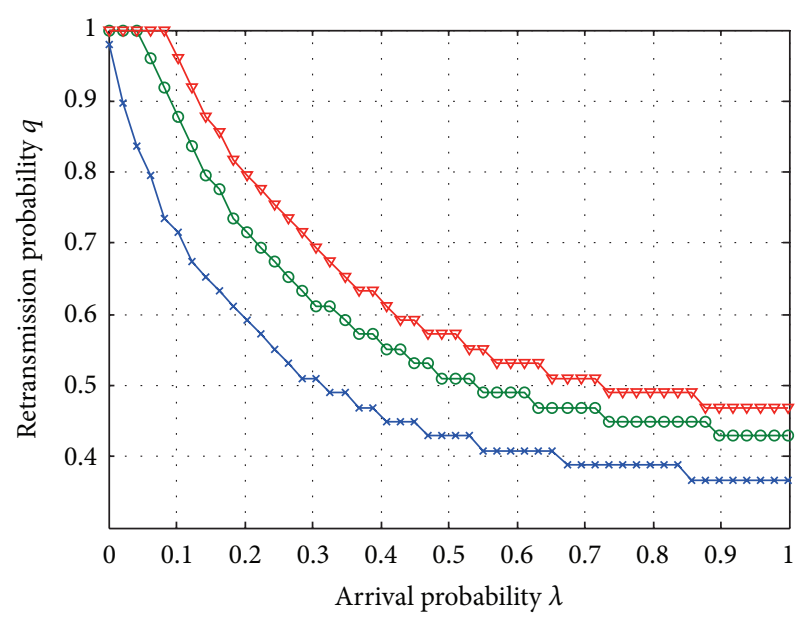

(a)

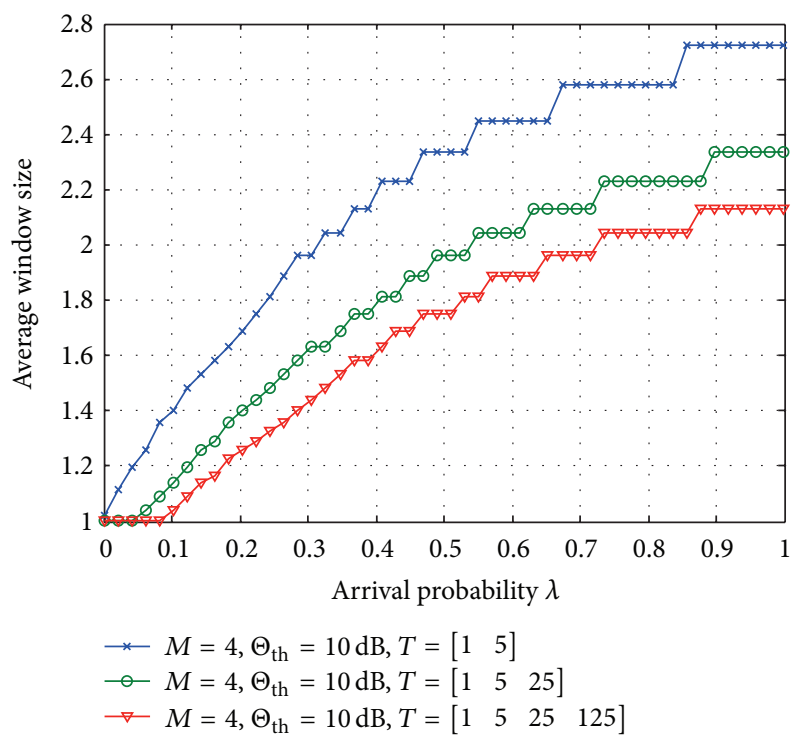

(b)

FIGURE 4: Impact of the number $N$ of available power levels on optimal retransmission probability and average window size for $M=4$ mobile stations.

arrival probability. Our results show that the use of an increasing number of power levels results in great benefits; see Figure 5. First, the MSs are not forced to drastically reduce the retransmission probability, resulting in a narrower window size. Furthermore, as a result of using a larger number of power levels, the network throughput is substantially higher than when using a lower number of different power levels; and the delay experienced by the stations is drastically reduced, especially at higher loads, that is, under high arrival probabilities. The results can be explained by the fact that the BS will have a higher chance of decoding correctly the received signal. As seen in Figure 5, we have also included the results of two other cases considering the use of five and six different power levels $T=[1,5,25,125,1000] \mathrm{mW}(N=5)$ and $T=[1,5,25,125,1000,1500] \mathrm{mW}(N=6)$. As seen from the figure, the use of a higher number of power levels does not 


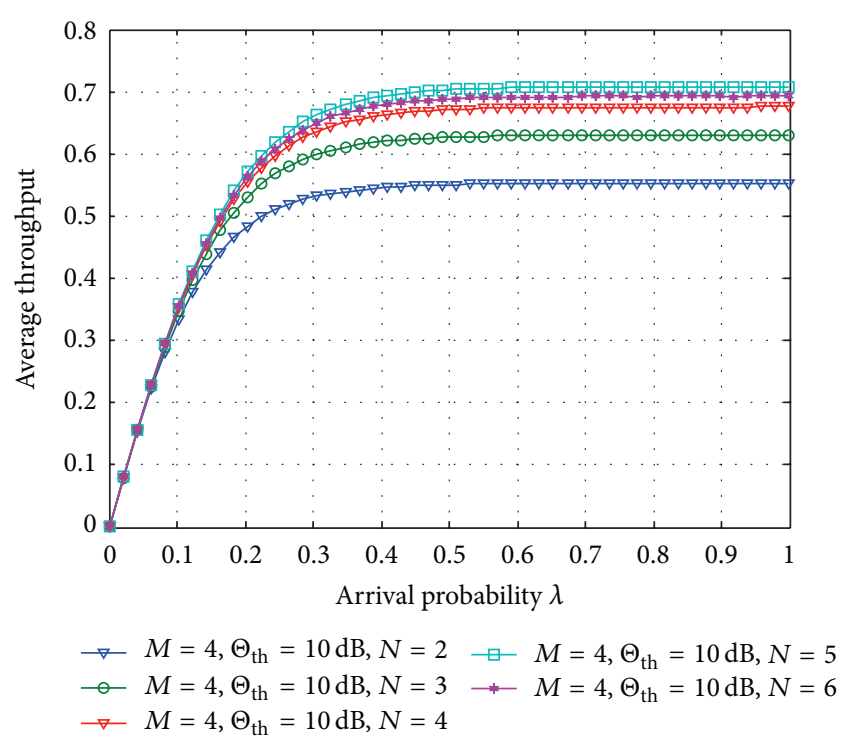

(a)

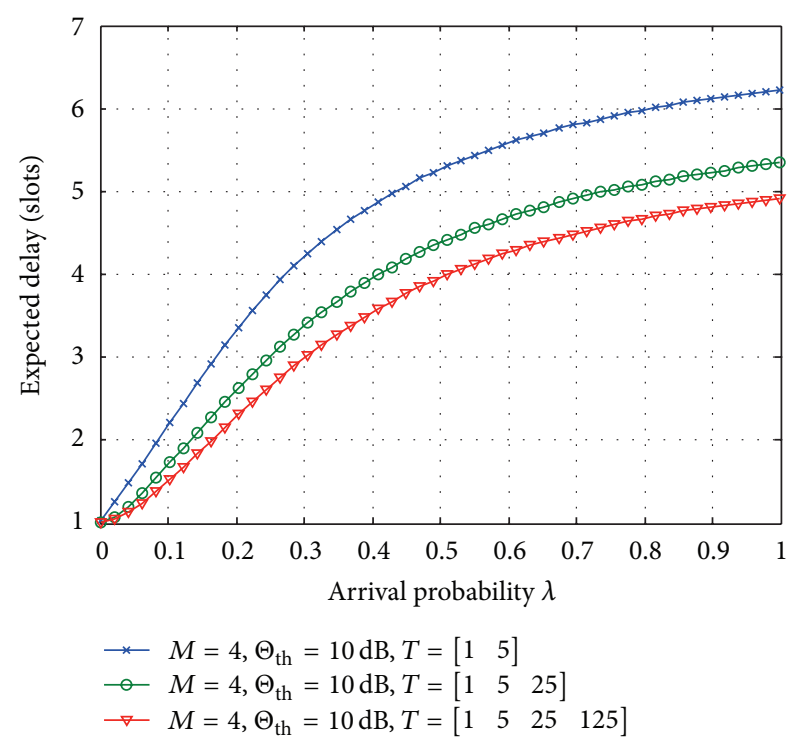

(b)

Figure 5: Impact of the number $N$ of available power levels on average throughput and expected delay for $M=4$ mobile stations.

result in a representative benefit on the performance of the proposed scheme.

5.1.3. Target SINR $\Theta_{t h}$. We now evaluate the performance of the system by varying the threshold SINR $\Theta_{\text {th }}$. As already stated, we have set $\sigma^{2}$ to zero, since its value will very much depend on the environmental conditions. Figures 6 and 7 show the retransmission probabilities, the average window size, the average throughput, and the expected delay, respectively, as function of arrival probability. We notice that the system performance deteriorates as $\Theta_{\text {th }}$ increases. Setting $\Theta_{\text {th }}$ to a higher value makes less likely the threshold to be achieved by the stations SINR values, reducing the probability

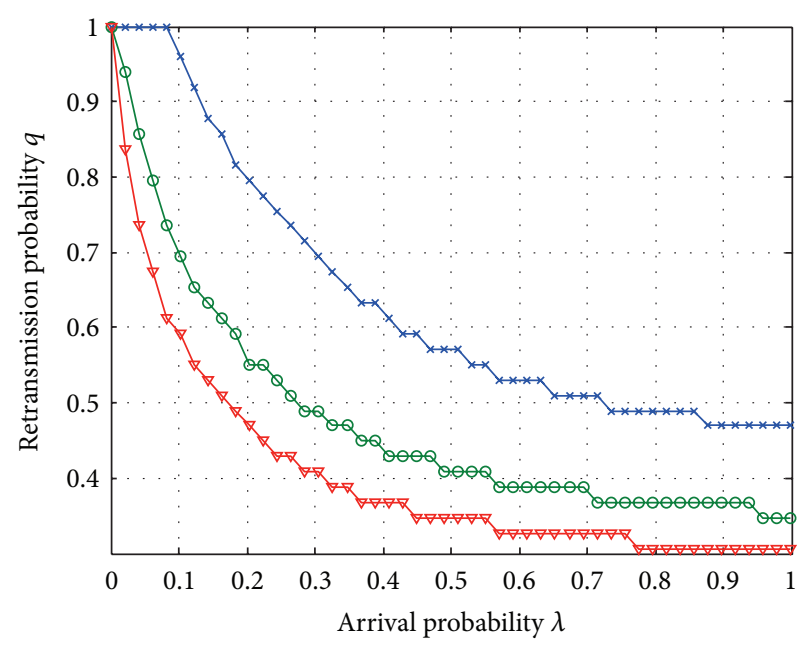

(a)

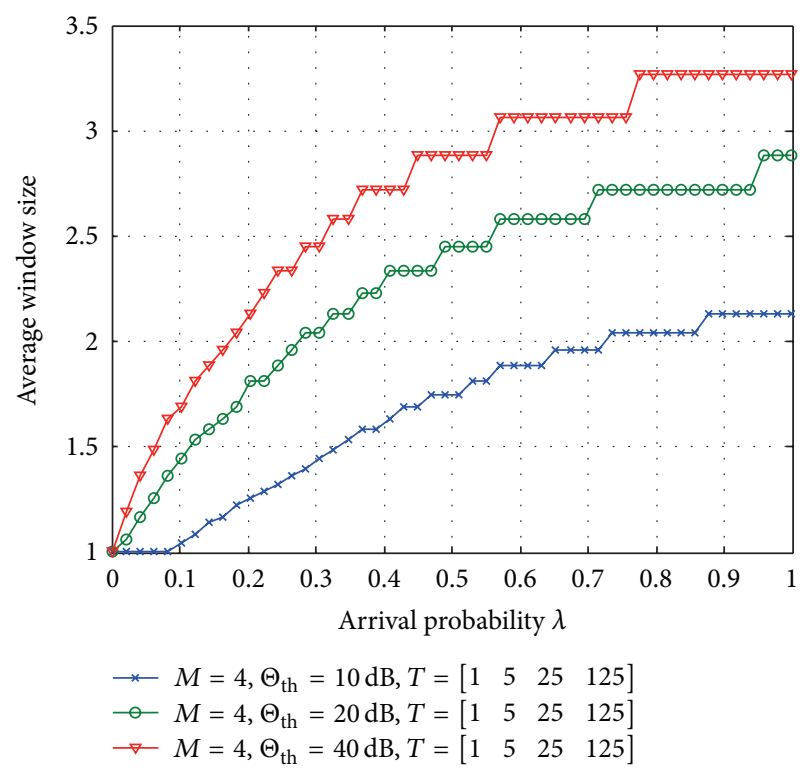

(b)

FIGURE 6: Impact of target SINR $\Theta_{\text {th }}$ on optimal retransmission probability and average window size for $M=4$ mobile stations.

of the capture effect to take place. On the contrary, a lower $\Theta_{\text {th }}$ increases the chances of the capture effect. However, the use of a lower SINR value in the presence of noise will result in a higher error probability on the decoding process. Our results show the great benefits of using MPL-WRA in environments, where values of the power levels of $\sigma^{2}$ remain relative low.

5.1.4. Power Levels Selection Distribution. Herein, we consider three different power selection policies: (1) equally or uniform probable, UPL; (2) higher priority on the selection of higher values, HPL, and (3) lower values are most likely to be selected, LPL. The main aim of this part of our study is to show that the probability distribution $X=\left[x_{1}, x_{2}, \ldots, x_{N}\right]$ used to select the transmit power has not a major impact on the performance of the proposed mechanism. As shown 


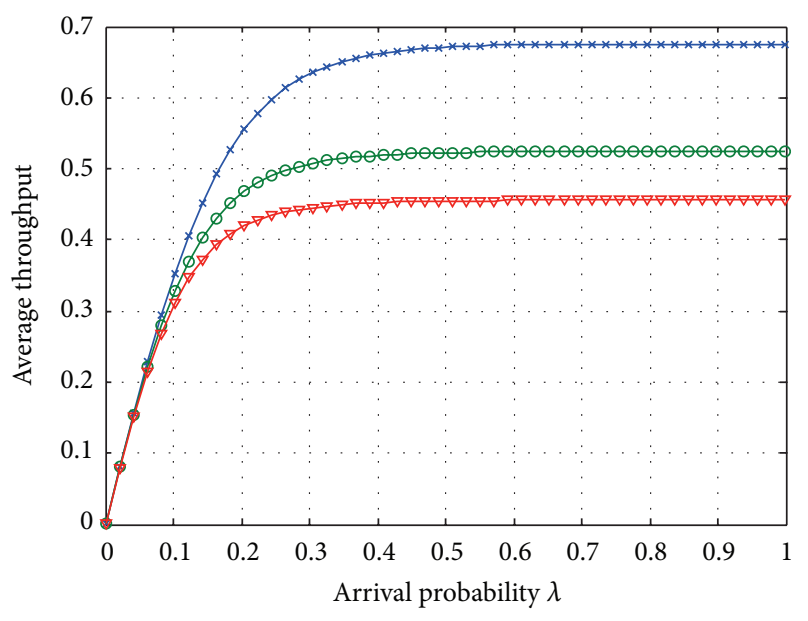

(a)

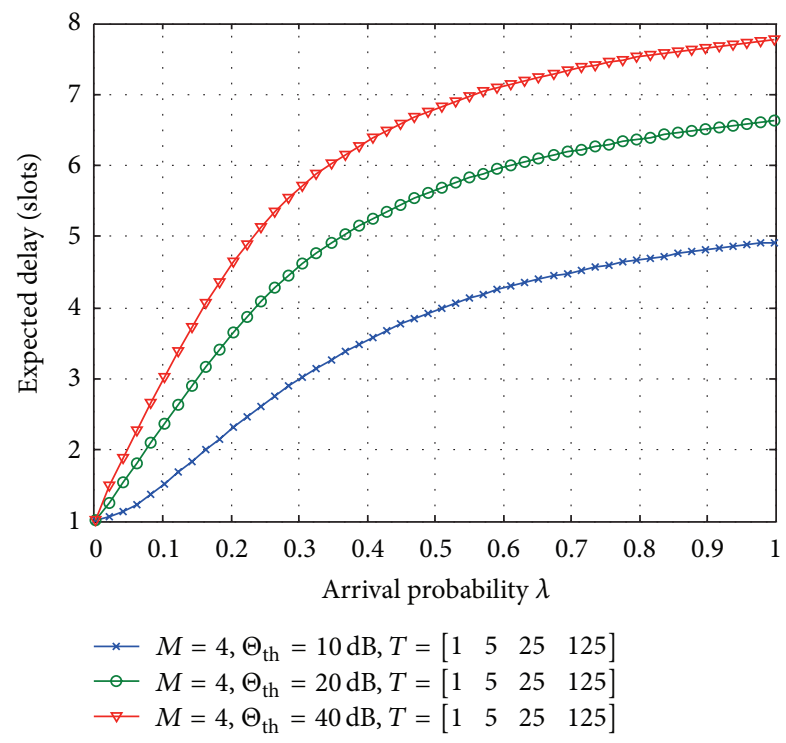

(b)

FIGURE 7: Impact of target SINR $\Theta_{\text {th }}$ on average throughput and expected delay for $M=4$ mobile stations.

in Figure 9, the uniform distribution UPL (i.e., $x_{i}=1 / N$ ) provides slightly better performance results; that is, prioritizing the selection of certain power levels over others does not have a significant impact on the network performance. When available power levels are uniformly distributed, they all have the same chance to be used. However, by favoring certain power levels over others, we push the system to behave as an equivalent system with fewer number of power levels available, which explains the observed impact on the network performance. An alternative explanation for performance degradation under HPL and LPL is that mobile stations become less aggressive with a less number of power levels available; see Figures 8 and 9.

5.2. Comparative Analysis of MPL-WRA and WRA. In this section, we carry out a comparative analysis of the wireless random access mechanism using power diversity with the

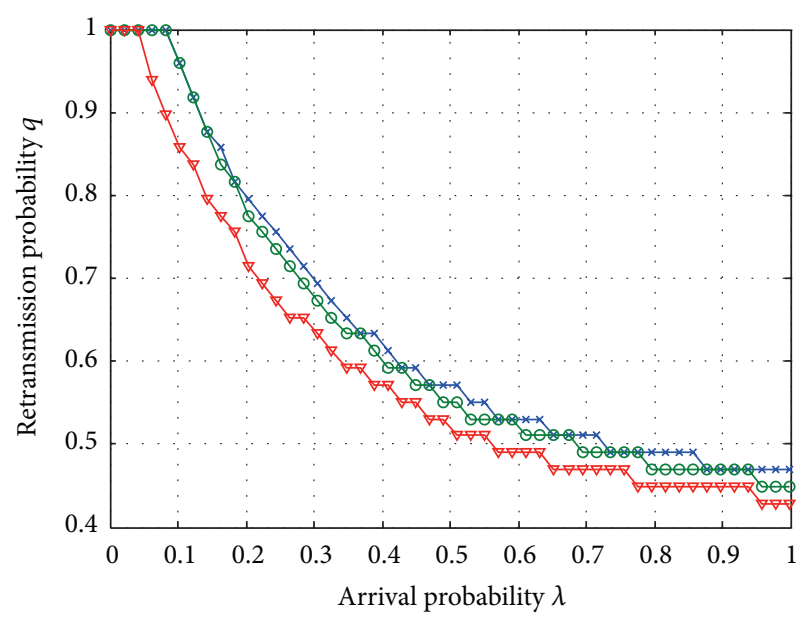

(a)

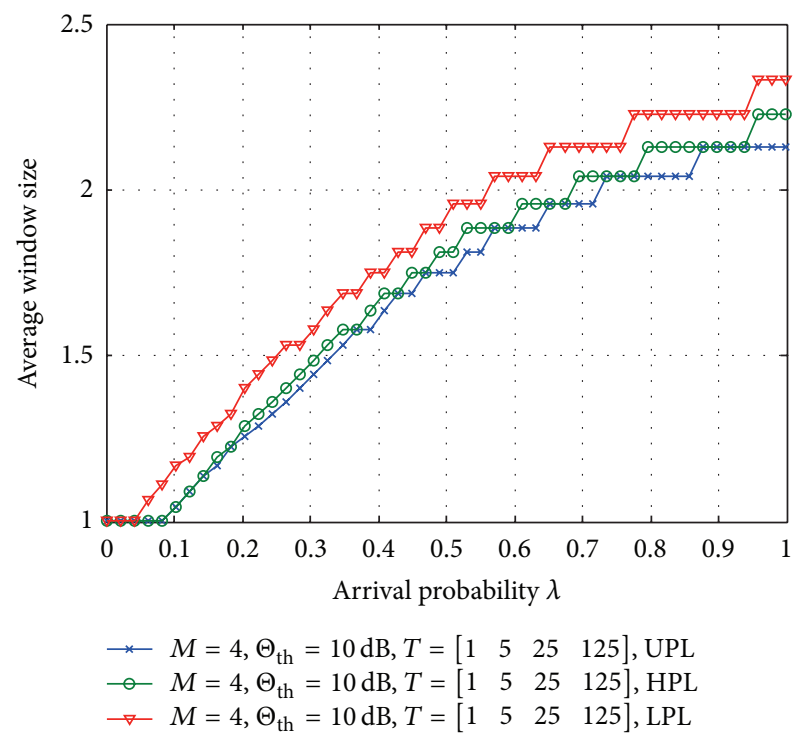

(b)

FIGURE 8: Optimal retransmission probability and optimal average window size under different power selection distributions.

single-power level version of it. Our main aim herein is to show the great benefits of properly setting the power diversity parameters over the use of a single-power level. In order to further show that our results are also applicable to larger network configurations, we also consider the case of a network consisting of $50 \mathrm{MSs}$. For this part of our study, we make use of the best setup configuration for MPL-WRA derived from the results obtained in the previous section. We therefore fix the threshold SINR to $\Theta_{\text {th }}=10 \mathrm{~dB}$ and consider the use of four power levels $T=[1,5,25,125] \mathrm{mW}$ selected following a uniform distribution probability $X=$ $[0.25,0.25,0.25,0.25]$. In order to show the scalability of our proposal, we consider two configurations representing a small-sized system ( $M=4 \mathrm{MSs})$ and a larger-sized system ( $M=50$ MSs).

Figures 10 and 11 show the optimal retransmission probability as a function of the arrival probability and the average 


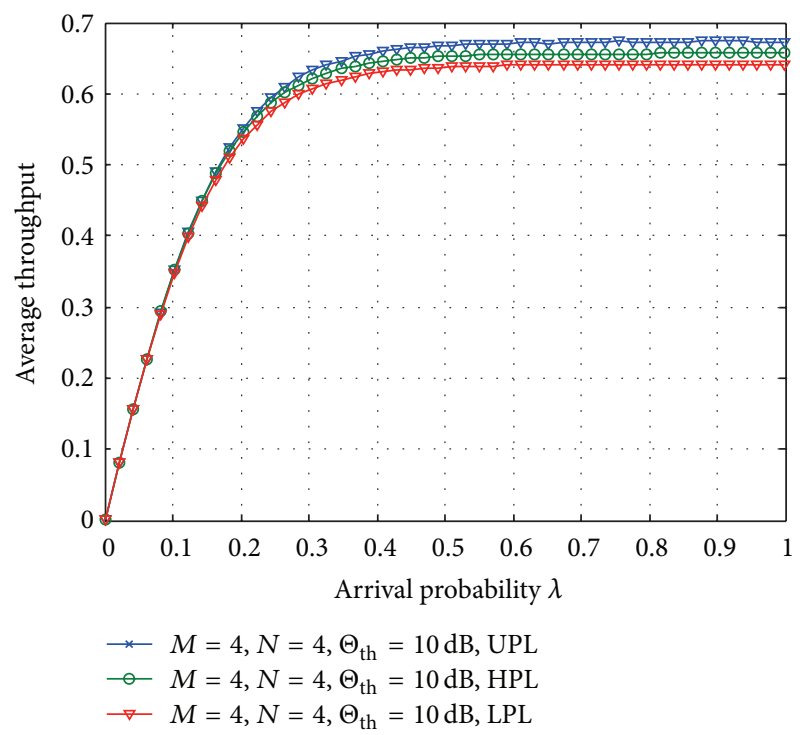

(a)

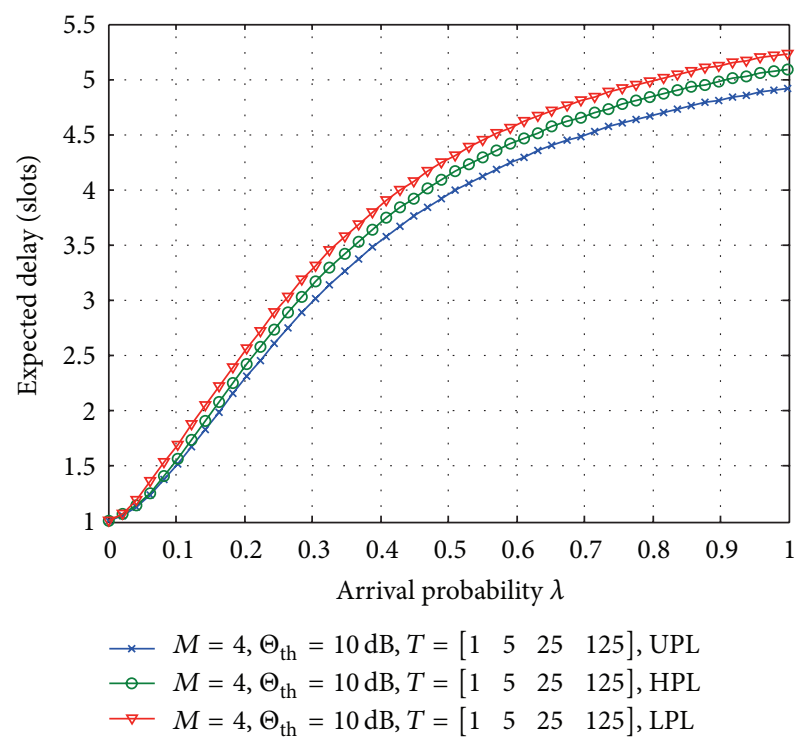

(b)

Figure 9: Average throughput and expected delay of bandwidth requests for $M=4$ mobile stations under different power selection distributions.

window size corresponding to the optimal retransmission probability for a fixed number of MSs. The relationship between these metrics is given by (15). From the figures, it is clear that MPL-WRA outperforms the WRA scheme. Even though that in a real setup, the size of the window will have to be set to a value expressed in terms of a power of 2; the results show that the proposed scheme could in fact be used in a practical setup. Take for instance the case of a system consisting of $50 \mathrm{MSs}$; the results show that the average window size could be set to 25 , that is, two to the power of five and not to two to the power of seven as required when using the single power WRA mechanism.

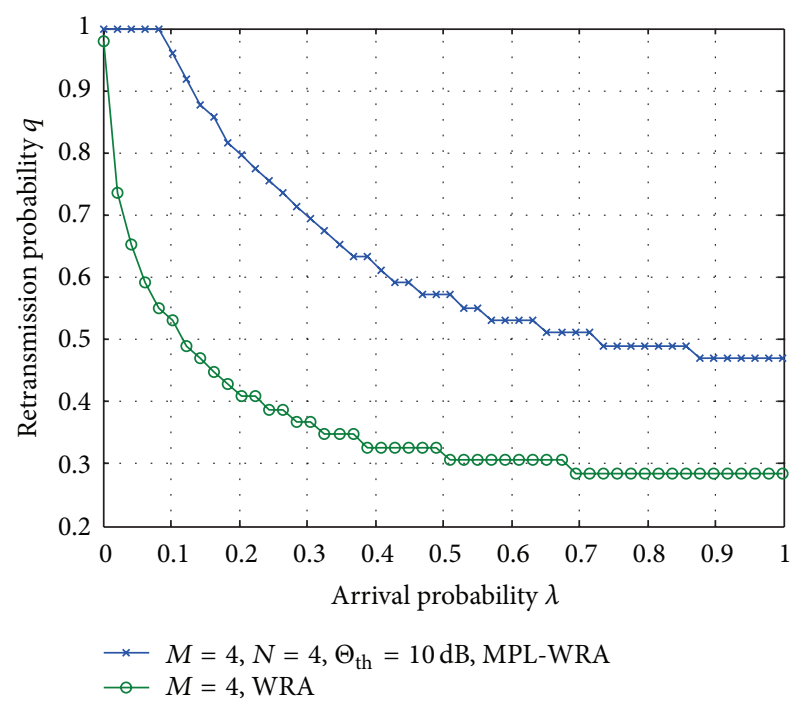

(a)

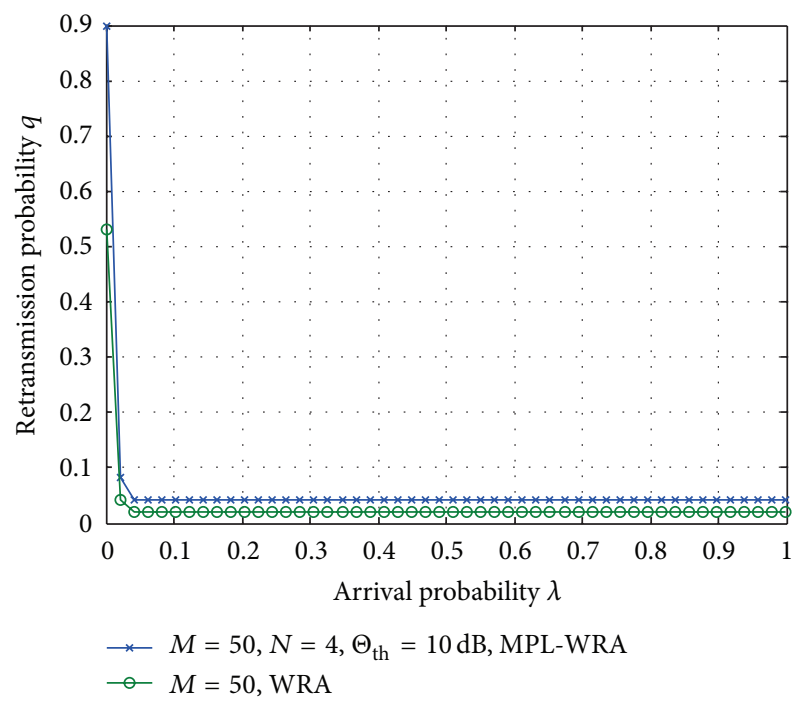

(b)

FIgURE 10: Optimal retransmission probability for $M=4$ and $M=$ 50 mobile stations.

Figures 12 and 13 show the average throughput and expected delay as a function of the arrival probability, for both mechanisms and the two system configurations under study, respectively. In general, for both schemes, we observe that the optimal retransmission probability as the arrival probability increases or the number of mobiles increases; that is, as the system becomes more congested (larger arrival probability), the transmission probability should be decreased as a counter measure to reduce the failure probability. The intuitive reason that the team optimal retransmission probabilities are close to 0 when arrival probabilities are close to one is that if an MS finds all other MSs backlogged, then it can transmit for very long time all its packets at a rate of a almost one packet per slot, without fearing collisions. Since its arrival probabilities are close to one, then throughput is not wasted during such periods. Note, however, that 


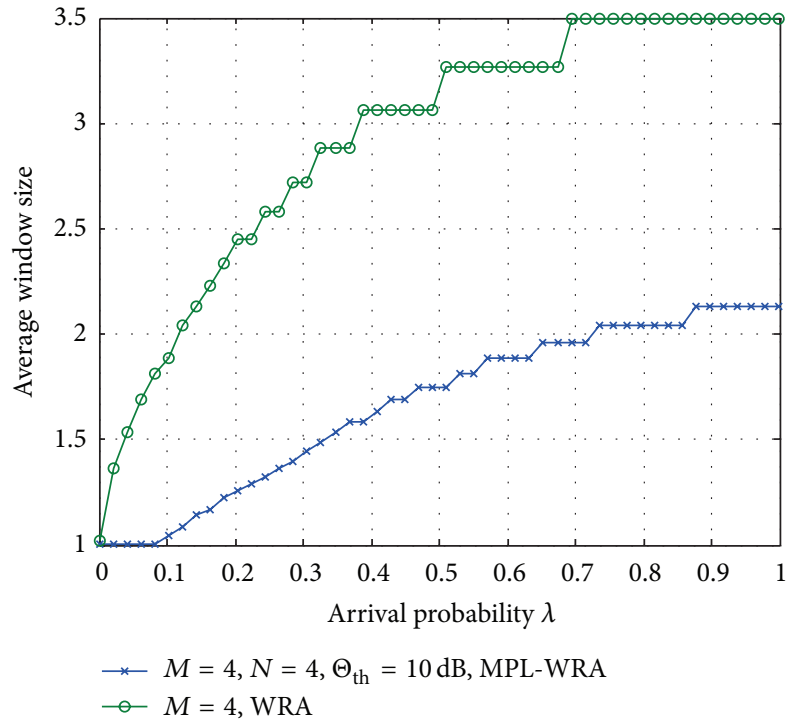

(a)

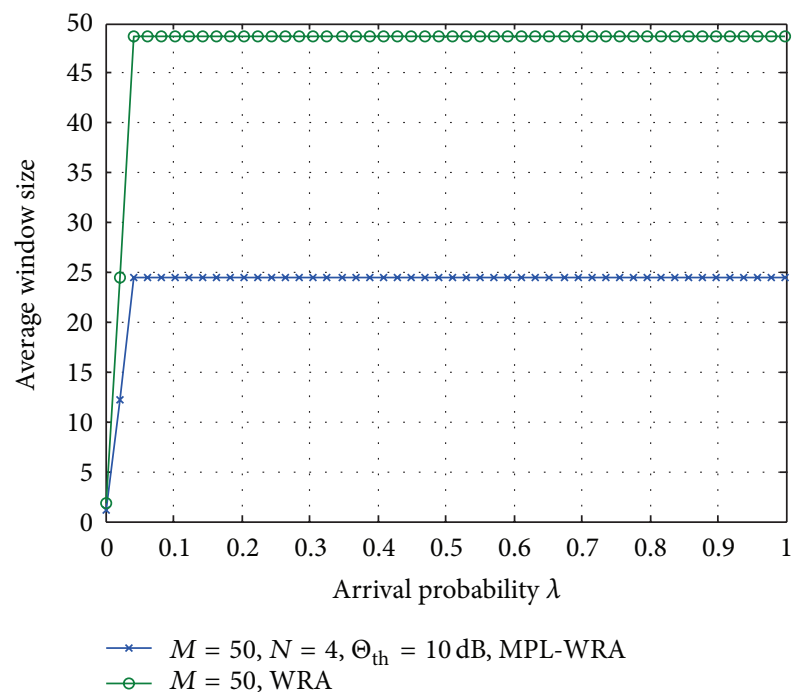

(b)

Figure 11: Optimal initial contention window for $M=4$ and $M=$ 50 mobile stations.

a throughput close to 1 cannot be achieved since with some nonnegligible probability, all MSs will be backlogged during long periods when retransmission probabilities are very low. Our results show that the multiple power mechanism, MPLWRA, outperforms the WRA scheme. In the case of a system consisting of $50 \mathrm{MSs}$, the proposed scheme results in an average normalized throughput of 0.60 , that is, an increase of more than $50 \%$ with respect to the throughput achieved by the single-power WRA protocol. The expected delay is also seen to be decreased, which gives some interesting and insightful uses of our scheme; that is, the use of MPL-WRA may prove useful for services having stricter constraints on delay.

Regarding the failure probability, Figure 14 shows the results for both schemes as a function of the arrival

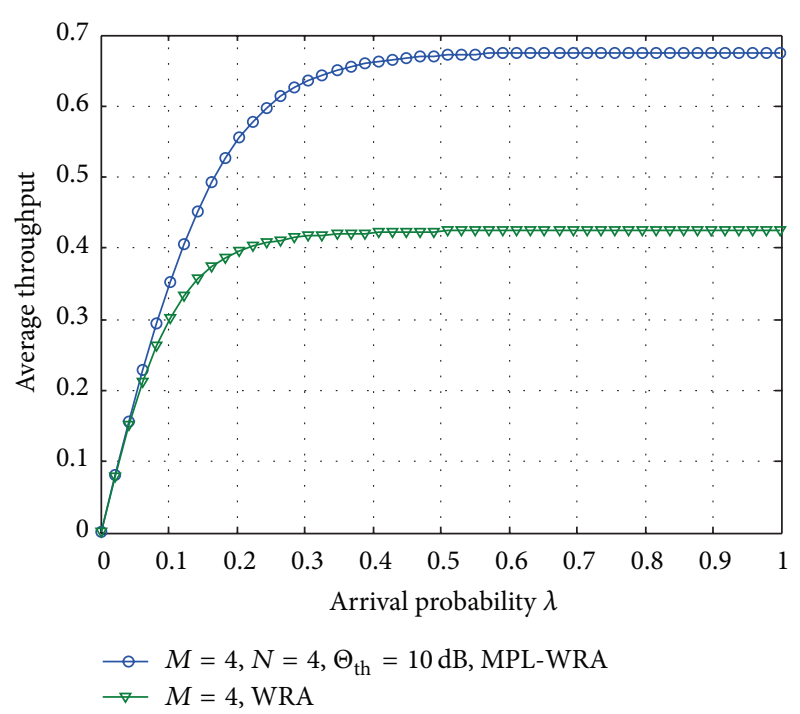

(a)

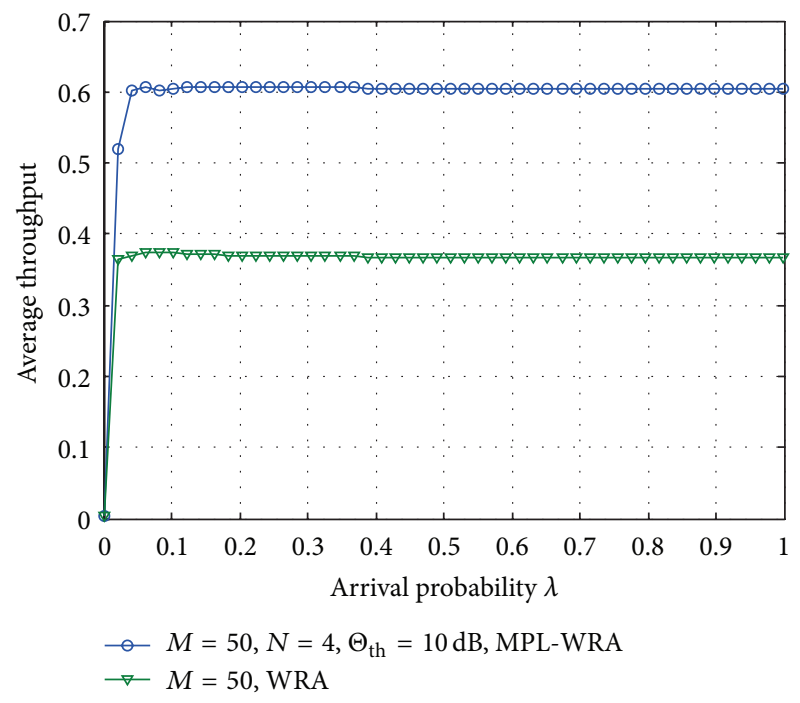

(b)

FIgURE 12: Average throughput for $M=4$ and $M=50$ mobile stations.

probability for $M=4$ MSs and $M=50$ MSs. Recall from the results in Figure 10 that our scheme encourages more aggressiveness in terms of channel access; that is, retransmission probabilities are higher for MPL-WRA than for WRA. However, our scheme greatly benefits from the capture effect. Figure 14(a) shows that the failure probability tends to be much higher for WRA than for MPL-WRA. The use of power diversity proves effective for boosting the performance of the WRA mechanism and it is only limited by the presence of accumulative noise (interference) caused by other adversarial MSs. Figure 14 shows that the failure probabilities increase as the arrival probability or the number of mobile station increases. An interesting feature is that in the case of a large-sized system, the failure probability tends to become constant for any offered load. 


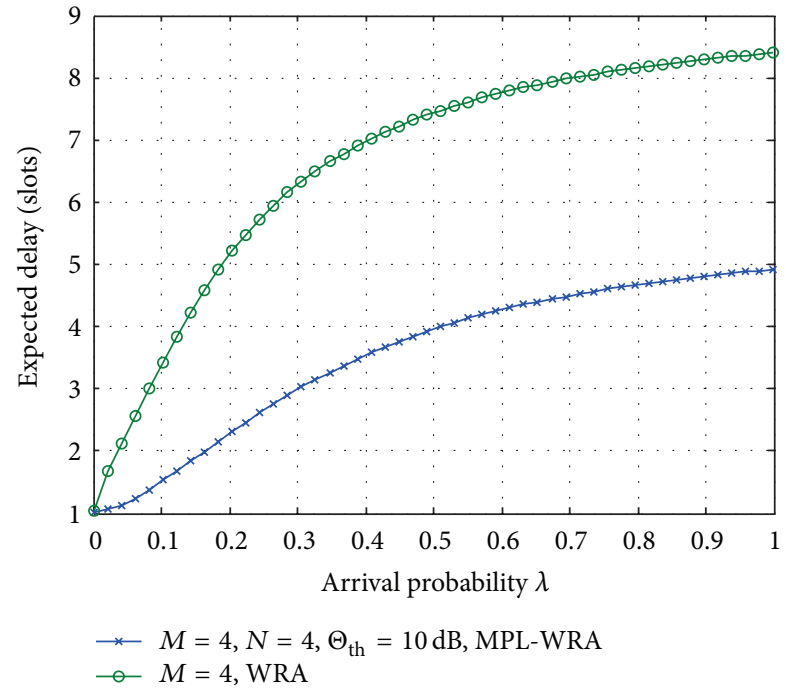

(a)

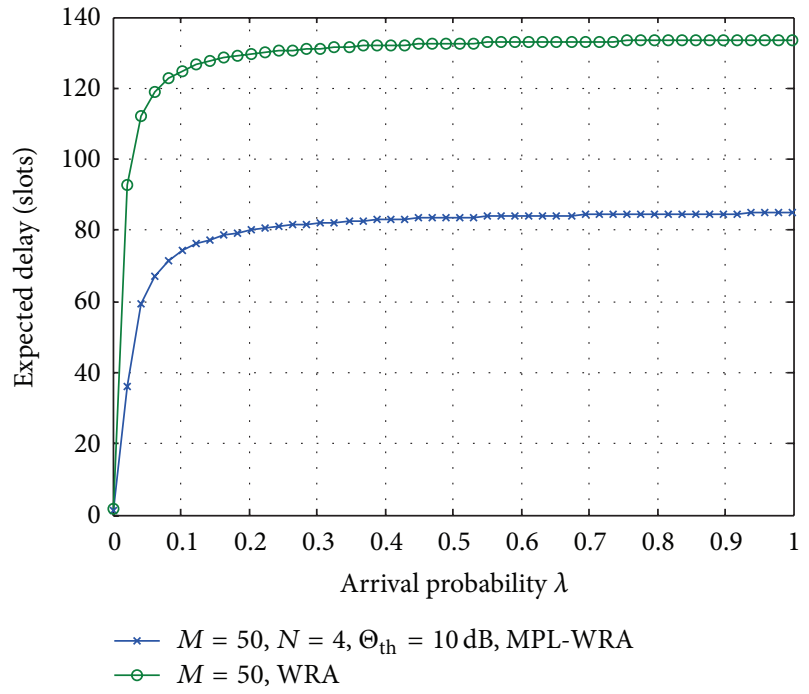

(b)

FIgURE 13: Expected delay for $M=4$ and $M=50$ mobile stations.

5.3. Performance of $M P L-R W A$ versus Network Size. In a real setup, it is most likely that the number of MSs will vary along; it is therefore worthwhile to explore the performance of our proposal as a function of the number of MSs. Towards this goal and based on our previous results, we set the system parameters to $T=[1,5,25,125] \mathrm{mW}(N=4)$ with $X=[0.25,0.25,0.25,0.25]$, and at three different values for the arrival probability $(0.1,0.5$, and 0.9$)$. The latter values correspond to a low, mild/average, and high activity MSs.

Figures 15 and 16 provide the optimal retransmission probability, the optimal average contention window size, the average throughput, and the expected delay, respectively. As expected, the retransmission probability decreases as the number of active MSs increases so as to counter expected collisions.

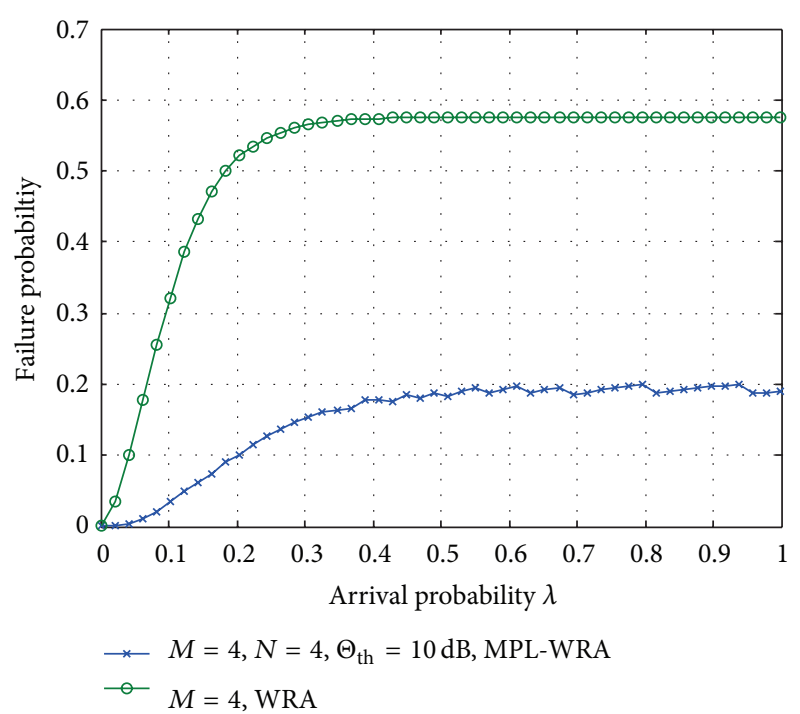

(a)

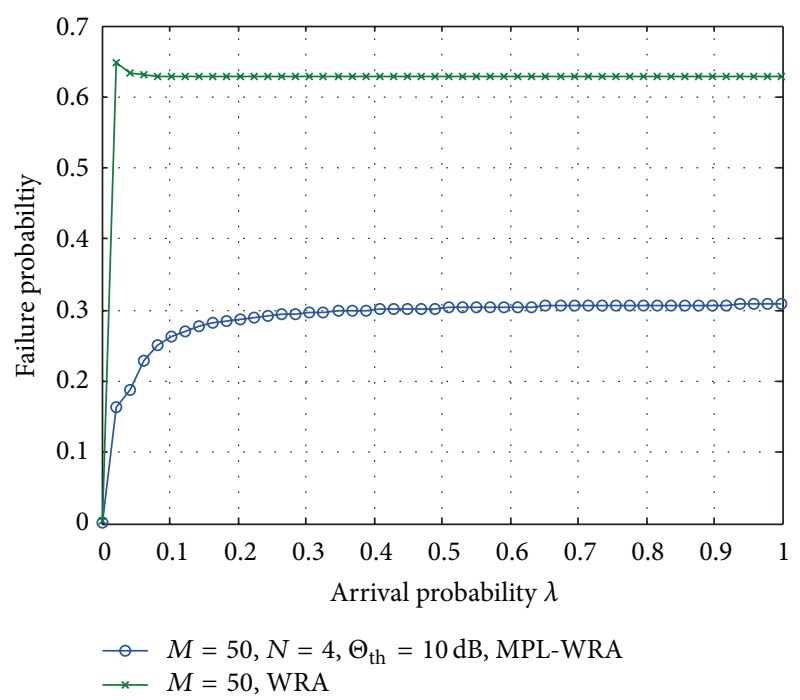

(b)

FIGURE 14: Failure probability for $M=4$ and 50 mobile stations versus the arrival rate $\lambda$.

Similar to our first set of results, the optimal throughput converges to 0.6 as the number of stations is increased. Note that since we are carrying out a joint optimization of the throughput and delay, the expected delay does not increase exponentially, but rather linearly as a function of the number of MSs. This can be explained by analyzing the form of expression (12) and by noticing that as more active MSs join the system the average throughput converges to a constant value, while the average number of ranging request $B(q)$ tends to grow linearly as a function of the number of active MSs.

In order to optimize the network throughput, the stations are required to reduce their retransmission probability in order to avoid more failure transmission. Even though this will result in a wider window size and an increase on the expected delay, the mechanism ensures that the network will maintain an effective throughput as high as $60 \%$. 


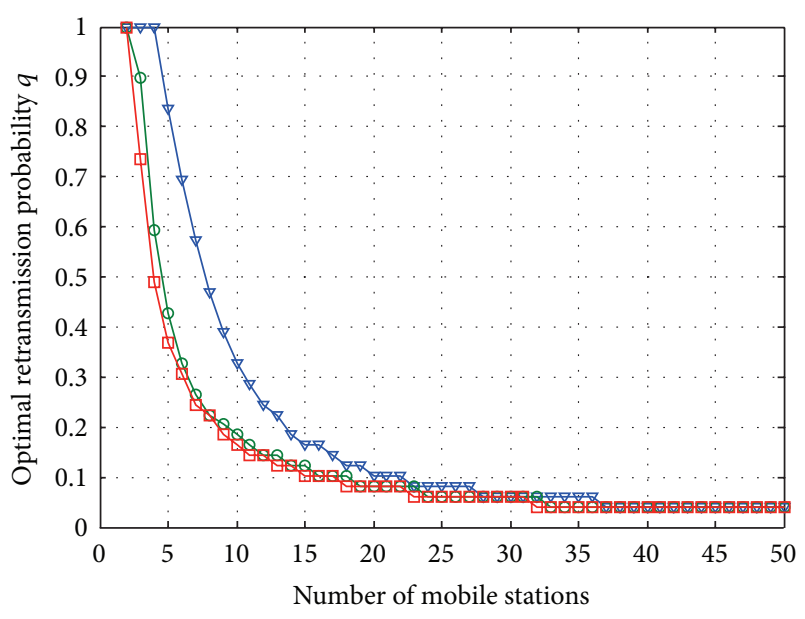

(a)

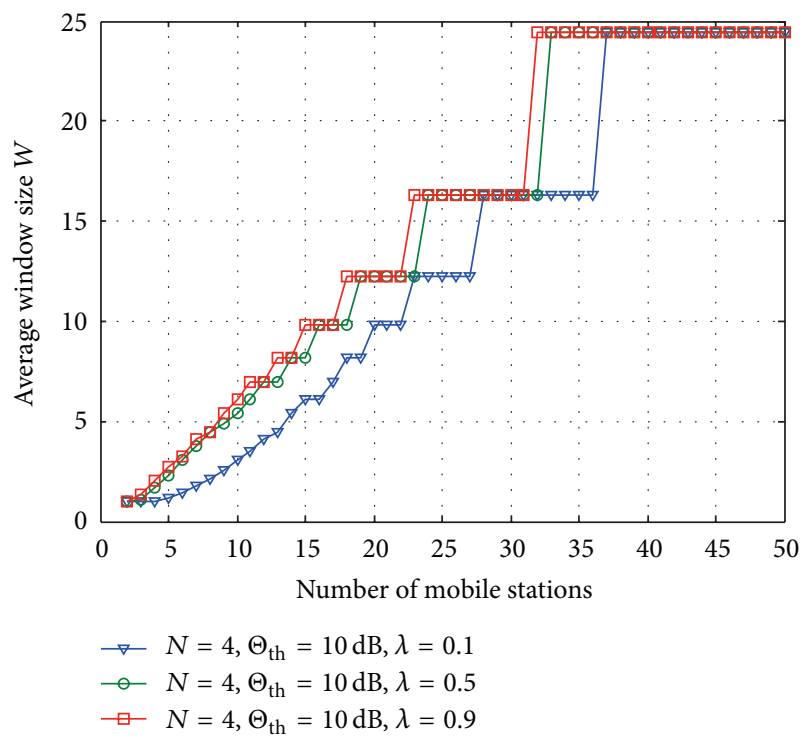

(b)

FIGURE 15: Optimal retransmission probability and optimal contention window size under different number of mobile stations.

\section{Concluding Remarks}

This paper presents MPL-WRA, a random access protocol for wireless networks using multiple power levels. We have built a Markovian model incorporating a power diversity scheme. Due to the capture effect, the base station may correctly decode the packet in the presence of collisions. Yet, accumulative signals corresponding to other simultaneous packets are simply considered as interference and therefore filtered out. When the instantaneous SINR is higher than the target threshold, a successful capture is declared. Function of the steady state vector and several performance metrics are derived, for instance, average throughput and expected delay. A detailed discussion on the existence of an optimal solution is provided. Under our algorithm, simulation results show that mobile stations are more aggressive, that is, transmit at higher probabilities. A quite paradoxical result is

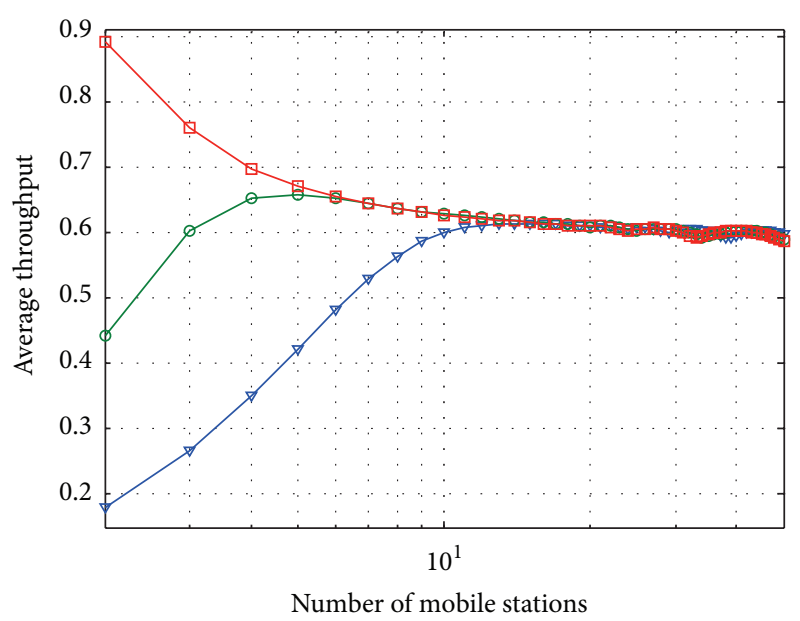

(a)

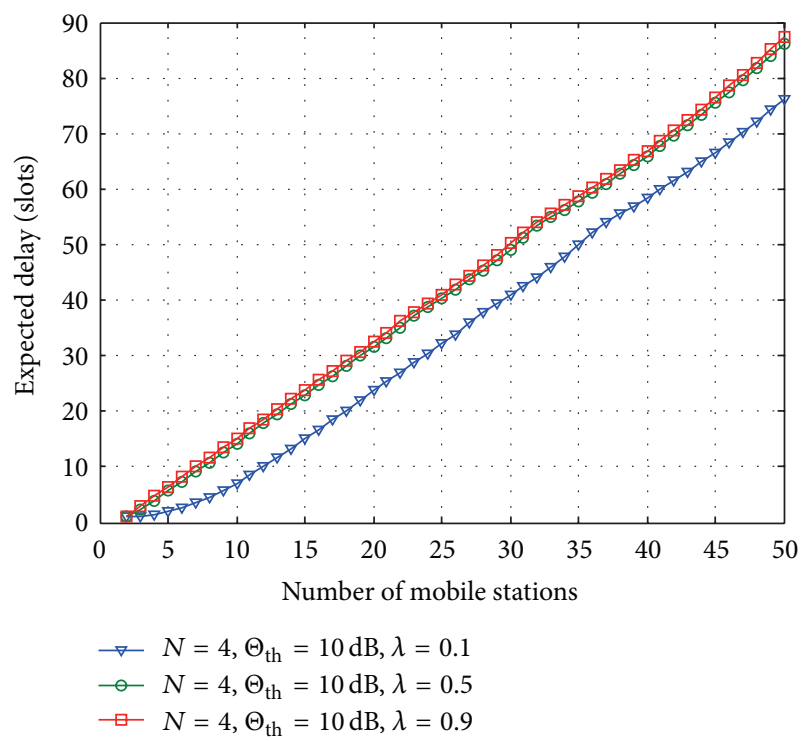

(b)

FIGURE 16: Average throughput and expected delay under different number of mobile stations.

that our MPL-WRA scheme encourages more transmissions. Intuitively, this may cause more concurrent access, but taking advantage from the capture effect, the average throughput and expected delay are both enhanced. In contrast to standard WRA where achievable bandwidth utilization is around 37\% (see [22]), MPL-WRA can achieve an upper bound of around $60 \%$.

We identify many research issues worth of further exploration. First, we are interested in exploring the noncooperative behaviour of mobile stations under the intervention of a leader, from game theory perspective. Second, we are interested in a strategic decoding of picked chunks, exploiting asynchrony across successive collisions such in ZigZag: a new iterative interference cancellation, where the algorithm attains the same throughput as if the colliding packets were a priori scheduled in separate time slots as described in [23]. Finally, we believe that MPL-WRA may have wider usage 
in wireless networking than what has been explored in this paper.

\section{Acknowledgments}

This work has been partially supported by the Spanish Ministry of Economy and Competitiveness (MINECO) and the European Commission (FEDER funds) under Grant no. TIN2012-38341-C04-04.

\section{References}

[1] E. Sabir, R. El-Azouzi, T. Jiménez, and E.-H. Bouyakhf, "Modeling slotted aloha as a stochastic game with random discrete power selection algorithms," Journal of Computer Systems, Networks, and Communications, vol. 2009, Article ID 572650, 28 pages, 2009.

[2] R. Jurdak, C. V. Lopes, and P. Baldi, "A survey, classification and comparative analysis of medium access control protocols for ad hoc networks?" IEEE Communications Surveys and Tutorials, vol. 6, no. 1, pp. 2-16, 2004.

[3] A. B. MacKenzie and S. B. Wicker, "Stability of slotted aloha with multi-packet reception and selfish users," in Proceedings of the IEEE INFOCOM, April 2003.

[4] R. R. Rao and A. Ephremides, "On the stability of interacting queues in a multiple-access system," IEEE Transactions on Information Theory, vol. 34, no. 5, pp. 918-930, 1988.

[5] W. Szpankowski, "Stability conditions for some distributed systems: buffered random access systems," Advances in Applied Probability, vol. 26, pp. 498-515, 1994.

[6] V. Anantharam, "The stability region of the finite-user slotted ALOHA protocol," IEEE Transactions on Information Theory, vol. 37, no. 3, pp. 535-540, 1991.

[7] D. J. Aldous, "Ultimate instability of exponential backoff protocol for acknowledgment based transmission control of random access communication channels," IEEE Transactions on Information Theory, vol. 33, no. 2, pp. 219-223, 1987.

[8] J. Goodman, A. G. Greenberg, N. Madras, and P. March, "Stability of binary exponential backoff," Journal of the ACM, vol. 35, no. 3, pp. 579-602, 1988.

[9] M. Paterakis, L. Georgiadis, and P. Papantoni-Kazakos, "On the relation between the finite and the infinite population models for a class of raa's," IEEE Transactions on Communications, vol. 35, no. 11, pp. 1239-1240, 1987.

[10] A. Haqiq, A. Karouit, and L. O. Barbosa, "A team study of the IEEE 802.16 collision resolution protocol," in Proceedings of the IFIP Wireless Days (WD '10), pp. 1-6, Venice, Italy, October 2010.

[11] A. Karouit, L. O. Barbosa, and A. Haqiq, "QoS criteria of the IEEE 802.16 collision resolution protocol," in Proceeding of International Conference MICS, Rabat, Morocco, 2010.

[12] E. Altman, D. Barman, A. Benslimane, and R. El Azouzi, "Slotted aloha with priorities and random power," in Proceedings of NETWORKING, pp. 610-622, Ontario, Canada, May 2005.

[13] J. H. Sarker, M. Hassan, and S. J. Halme, "Power level selection schemes to improve throughput and stability of slotted ALOHA under heavy load," Computer Communications, vol. 25, no. 18, pp. 1719-1726, 2002.

[14] B.-J. Kwak, N.-O. Song, and L. E. Miller, "Performance analysis of exponential backoff," IEEE/ACM Transactions on Networking, vol. 13, no. 2, pp. 343-355, 2005.
[15] J. Barcelo, B. Bellalta, C. Cano, and M. Oliver, "LearningBEB: avoiding collisions in WLAN," in Proceedings of the IFIP EUNICE, 2008.

[16] R. El-Azouzi, E. Sabir, S. K. Samanta, and R. El-Khoury, "Asymptotic delay analysis and timeout-based admission control for ad hoc wireless networks with asymmetric users," Computer Communications, vol. 33, no. 17, pp. 2057-2069, 2010.

[17] J. Yan and G.-S. Kuo, "Cross-layer design of optimal contention period for IEEE 802.16 BWA systems," in Proceedings of the IEEE International Conference on Communications (ICC '06), pp. 1807-1812, July 2006.

[18] D. Bertsekas and R. Gallager, Data Networks, Prentice Hall, Englewood Cliffs, NJ, USA, 1987.

[19] G. E. Andrews, The Theory of Partitions, Addison-Wesley, Advanced Book Program, Reading, Mass, USA, 1976.

[20] E. M. Reingold, J. Nievergelt, and N. Deo, Combinatorial Algorithms, Prentice Hall, Englewood Cliffs, NJ, USA, 1977.

[21] R. T. B. Ma, V. Misra, and D. Rubenstein, "Modeling and analysis of generalized slotted-aloha MAC protocols in cooperative, competitive and adversarial environments," in Proceedings of the 26th IEEE International Conference on Distributed Computing Systems (ICDCS '06), Lisbon, Portugal, July 2006.

[22] D. Chuck, K.-Y. Chen, and J. M. Chang, "A comprehensive analysis of bandwidth request mechanisms in IEEE 802.16 networks," IEEE Transactions on Vehicular Technology, vol. 59, no. 4, pp. 2046-2056, 2010.

[23] S. Gollakota and D. Katabi, "ZigZag decoding: combating hidden terminals in wireless networks," in Proceedings of SIGCOMM, Seattle, Wash, USA, 2008. 


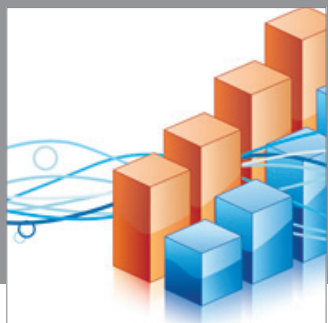

Advances in

Operations Research

mansans

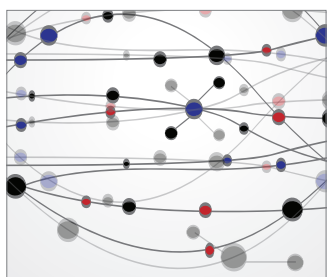

The Scientific World Journal
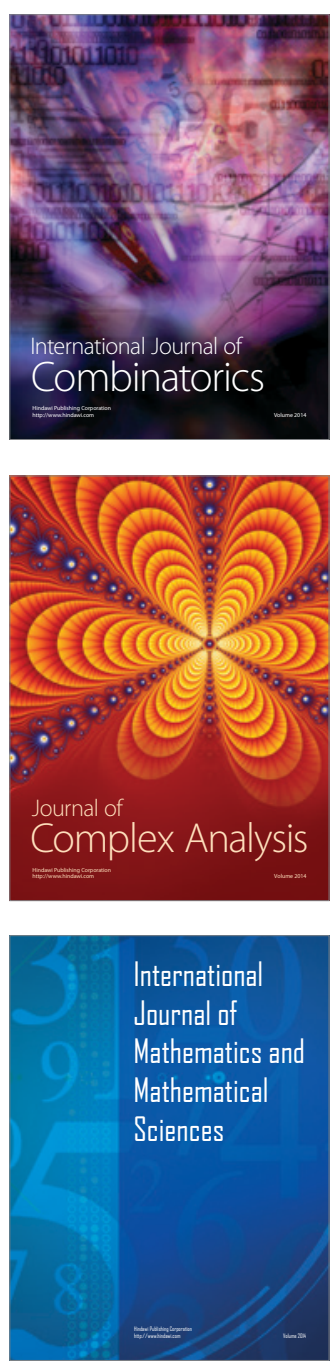
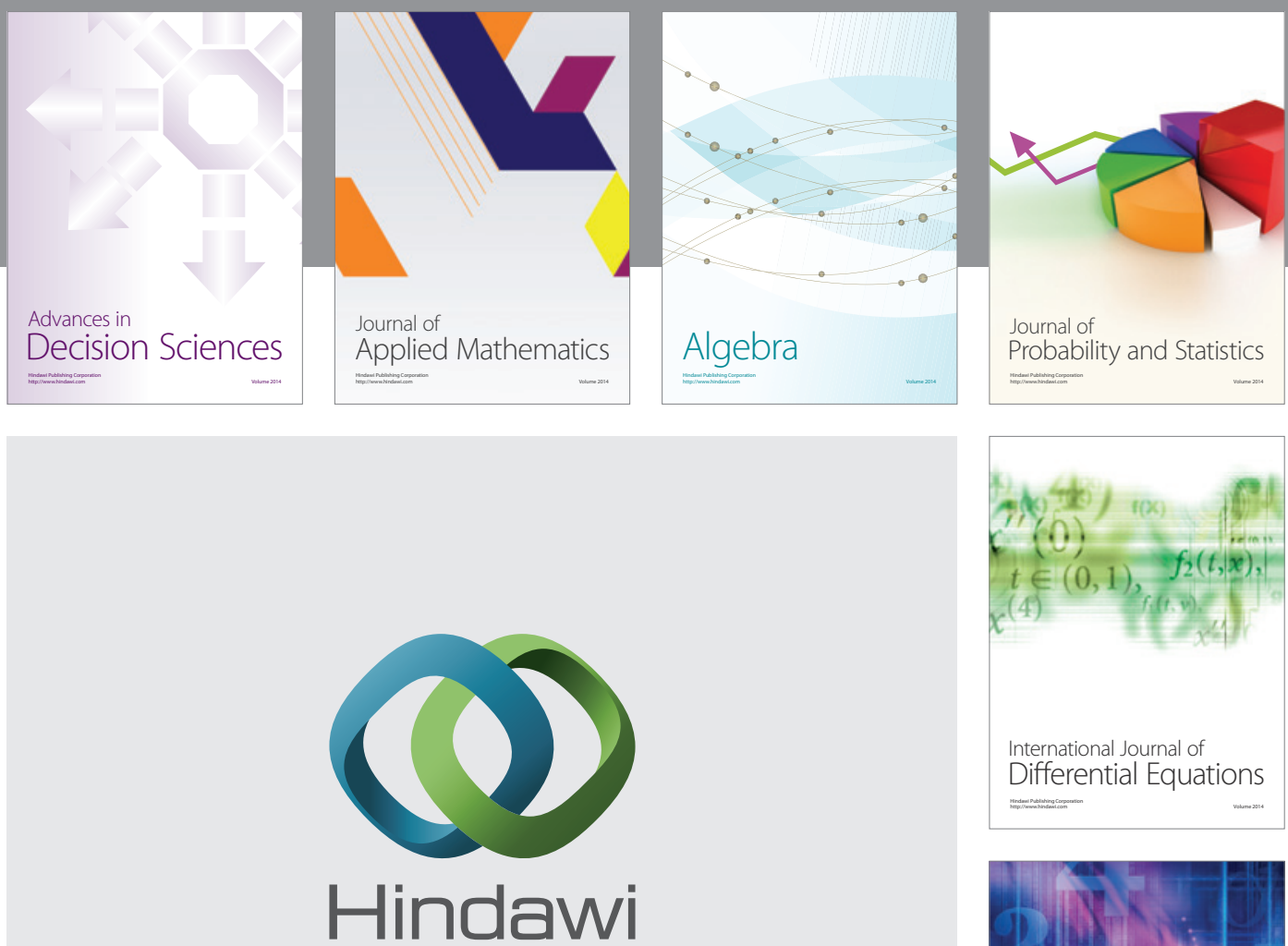

Submit your manuscripts at http://www.hindawi.com
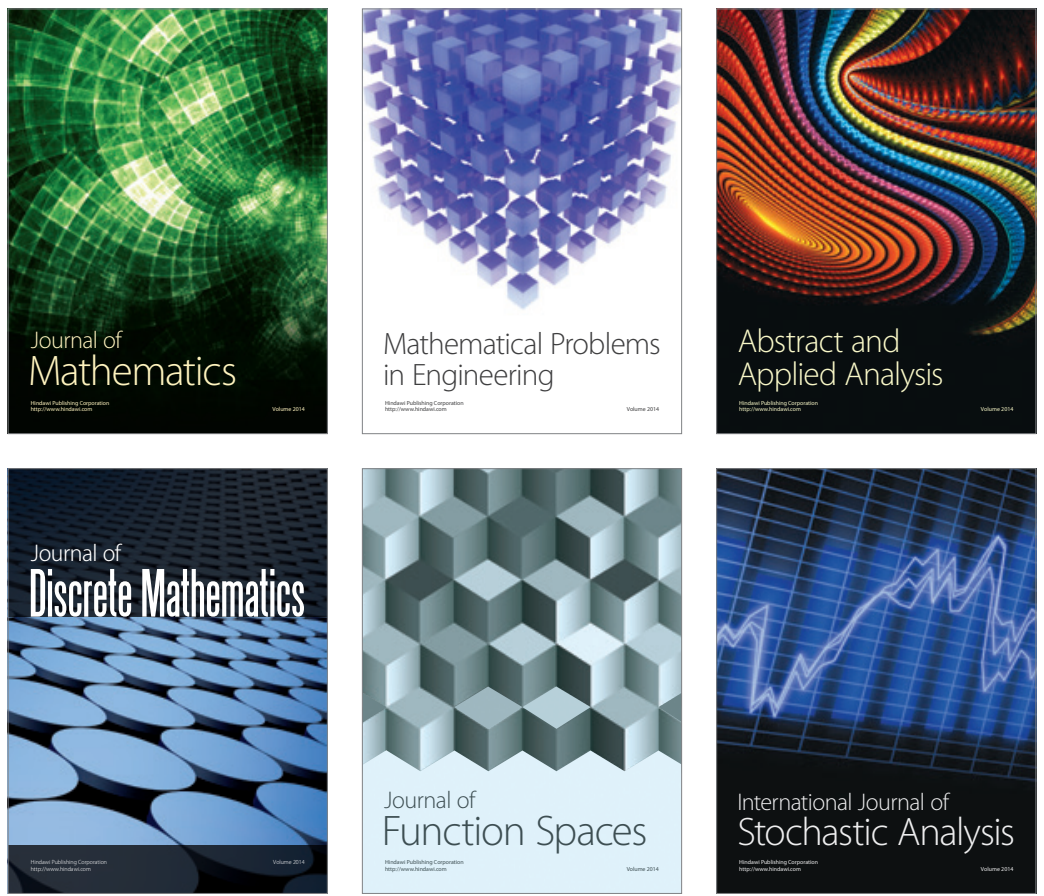

Journal of

Function Spaces

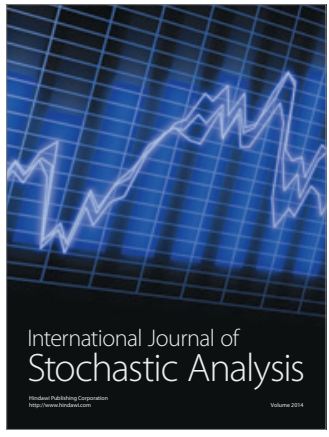

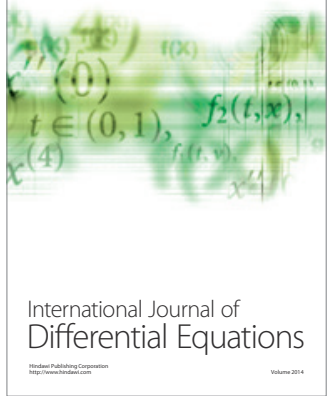
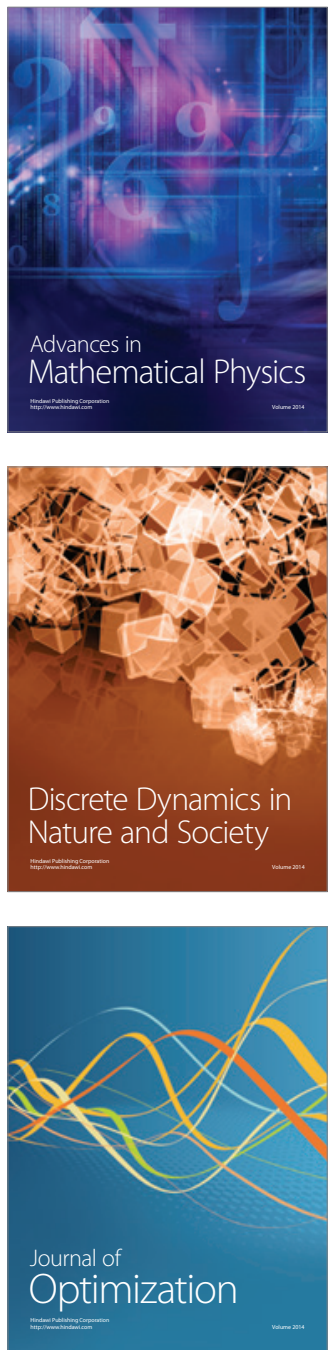